\title{
ABSOLUTELY CONTINUOUS SPECTRUM OF MULTIFREQUENCY QUASIPERIODIC SCHRÖDINGER OPERATOR
}

\author{
XUANJI HOU, JING WANG, AND QI ZHOU
}

\begin{abstract}
In this paper, we prove that for any $d$-frequency analytic quasiperiodic Schrödinger operator, if the frequency is weak Liouvillean, and the potential is small enough, then the corresponding operator has absolutely continuous spectrum. Moreover, in the case $d=2$, we even establish the existence of ac spectrum under small potential and some super-Liouvillean frequency, and this result is optimal due to a recent counterexample of Avila and Jitomirskaya [8].
\end{abstract}

\section{INTRODUCTION AND MAIN RESULTS}

This work is concerned with one-dimensional analytic Schrödinger operators $H_{v, \alpha, \phi}$ defined on $l^{2}(\mathbb{Z})$

$$
\left(H_{v, \alpha, \phi} u\right)_{n}=u_{n-1}+u_{n+1}+v(\phi+n \alpha) u_{n},
$$

where $v \in C^{\omega}\left(\mathbb{R}^{d} / \mathbb{Z}^{d}, \mathbb{R}\right), \phi \in \mathbb{R}^{d} / \mathbb{Z}^{d}=: \mathbb{T}^{d}$ and $\alpha \in \mathbb{R}^{d}$ is rational independent, i.e., $\langle\mathbf{k}, \alpha\rangle \notin \mathbb{Z}$ for all non-zero $\mathbf{k} \in \mathbb{Z}^{d}$. We recall that $v, \phi$, and $\alpha$ are the potential, the phase and the frequency respectively. Because of its strong connection with physics, analytic quasiperiodic Schrödinger operators have been fruitfully studied for a long time. We invite the reader to consult the recent surveys $[16,25]$ and the references therein.

The spectrum set of $H_{v, \alpha, \phi}$ is a compact set of $\mathbb{R}$, independent of the phase $\phi$, since the base dynamics $\phi \mapsto \phi+\alpha$ is minimal, and we will denote it by $\Sigma(v, \alpha)$ for short. The spectral measure of $H_{v, \alpha, \phi}$ can be decomposed into atomic, singular continuous and absolutely continuous (ac) parts, while ac part corresponds to the strongest transport properties. The ac spectrum part of $H_{v, \alpha, \phi}$ is also independent of the phase $\phi$ [29], and we use $\Sigma_{a c}(v, \alpha)$ to denote it. We are most interested in when $\Sigma_{a c}(v, \alpha) \neq \varnothing$. The well-known Kotani's theory $[26,30]$ tells us that $\Sigma_{a c}(v, \alpha)$ has a close relationship with the Lyapunov exponent

$$
L(\alpha, E-v)=\lim _{n \rightarrow \infty} \frac{1}{n} \int_{\mathbb{R}^{d} / \mathbb{Z}^{d}} \ln \left\|S_{E-v}^{(n)}(\phi)\right\| d \phi \geq 0,
$$

Date: April 10, 2020. 
where $E \in \mathbb{R}$ is the energy and

$$
S_{E-v}^{(n)}(\phi)=\left(\begin{array}{cc}
E-v(\phi+(n-1) \alpha) & -1 \\
1 & 0
\end{array}\right) \cdots\left(\begin{array}{cc}
E-v(\phi) & -1 \\
1 & 0
\end{array}\right) .
$$

Let $\Sigma_{0}(v, \alpha)=\{E \in \mathbb{R} \mid L(\alpha, E-v)=0\}$. Then $\Sigma_{a c}(v, \alpha)$ is the essential closure $^{1}$ of $\Sigma_{0}(v, \alpha)[26,30]$.

In order to study the spectral property of $H_{v, \alpha, \phi}$, it is very useful to introduce the coupling constant $\lambda$ in front of the potential $v$. By the symmetry, we just assume $\lambda>0$. If the coupling constant $\lambda$ is sufficiently large, then $L(\alpha, E-\lambda v)>0$ for any $E \in \mathbb{R}[11,14,22,23,31]$. Therefore by Kotani's Theory, there is no hope to find $\Sigma_{a c}(\lambda v, \alpha)$ of $H_{\lambda v, \alpha, \theta}$ for large coupling constant.

If the coupling constant $\lambda$ is sufficiently small, there is a tendency of occurrence of ac spectrum, where the arithmetic properties of the frequency come into play. We recall that $\alpha$ is Diopantine (denote it by $\alpha \in D C$ ), if there exist $\gamma, \tau>0$, such that for any $\mathbf{k} \in \mathbb{Z}^{d} \backslash\{0\}$

$$
\|\langle\mathbf{k}, \alpha\rangle\|_{\mathbb{R} / \mathbb{Z}}=\min _{j \in \mathbb{Z}}|\langle\mathbf{k}, \alpha\rangle-j| \geq \frac{\gamma}{|\mathbf{k}|^{\tau}} .
$$

Based on KAM method, Dinaburg-Sinai [17] proved that $\Sigma_{a c}(\lambda v, \alpha) \neq \varnothing$ in the perturbative small regime $\lambda<\lambda_{0}$. Here perturbative means that $\lambda_{0}$ depends on $\alpha$ through the Diophantine constants $\gamma, \tau$. Also under the same assumption, Eliasson [19] showed that in fact we have $\Sigma(\lambda v, \alpha)=\Sigma_{a c}(\lambda v, \alpha)$.

Moreover, in the case of $d=1$, one can even anticipate some nonperturbative results. Making use of the specificity of one frequency, some new elaborate techniques are developed to prove some sharp results. As $\alpha \in D C$, based on non-perturbative Anderson localization result, AvilaJitomirskaya [7] proved that there exists $\lambda_{1}$ which does not depend on $\alpha$, such that $\Sigma(\lambda v, \alpha)=\Sigma_{a c}(\lambda v, \alpha)$ as $\lambda<\lambda_{1}$. Recently, Avila-Fayad-Krikorian [6] and Hou-You [24] independently developed non-standard KAM techniques, showed that $\Sigma_{a c}(\lambda v, \alpha) \neq \varnothing$ for $\lambda<\lambda_{1}(v)$ and for any irrational $\alpha$. Indeed, Avila [3, 4] was able to show that $\Sigma(\lambda v, \alpha)=\Sigma_{a c}(\lambda v, \alpha)$.

If the coupling constant $\lambda$ is neither too large nor too small, which is the so-called global case, the problem is extremely complicated, since few methods can be successfully applied in obtaining detailed informations of the spectrum. Nevertheless, in the one frequency case, we would like to highlight Avila's fascinating global theory of analytic Schrödinger operators [2] and his well-known spectral dichotomy conjecture(now proved by himself in $[3,4])$. He shows that for typical analytic one-frequency Schrödinger operator, it doesn't have singular continuous spectrum. This is an already very deep result from the ac spectrum side, as was former pointed out by Avila and Krikorian [9], $\Sigma_{0}(\lambda v, \alpha)$ may support non-ac spectrum. Avila's

\footnotetext{
${ }^{1}$ Given a set $A \subseteq \mathbb{R}$, the essential closure of $A$ is defined as the set $\{E \in \mathbb{R} \mid \forall \epsilon>0, A \cap(E-\epsilon, E+\epsilon)$ is of positive Lebesgue measure $\}$.
} 
global theory $[3,4]$ thus establishes the deep relations between the existence of ac spectrum and the vanishing of the Lyapunov exponent in a band [2].

1.1. Multifrequency Schrödinger operator. However, not to mention Avila's global theory can't be generalized to the multifrequency case [18], one can even not anticipate non-perturbative results for the multifrequency case (a counter example is given by Bourgain [10]). Comparing to the one frequency case, as pointed by Eliasson [20]: "while almost everything is known for one frequency case, almost everything is unknown for multifrequency case".

In this paper, we establish two results which confirm the existence of ac spectrum for multifrequency quasiperiodic Schrödinger operator beyond the Diophantine frequency. Before introducing them, we need to give some necessary definitions.

For $\tilde{\alpha} \in \mathbb{R} \backslash \mathbb{Q}, \alpha^{\prime} \in \mathbb{T}^{d-1}$, we say that the pair $\left(\tilde{\alpha}, \alpha^{\prime}\right)$ is weak Liouvillean, if there exist $\gamma, \tau>0,0<\tilde{U}<\infty$, such that

$$
\begin{gathered}
\widetilde{U}=\widetilde{U}(\tilde{\alpha})=: \sup _{n>0} \frac{\ln \ln \tilde{q}_{n+1}}{\ln \tilde{q}_{n}}, \\
\left\|k \tilde{\alpha}+\left\langle l, \alpha^{\prime}\right\rangle\right\|_{\mathbb{R} / \mathbb{Z}} \geq \frac{\gamma}{(|k|+|l|)^{\tau}} \text { for } k \in \mathbb{Z}, l \in \mathbb{Z}^{d-1} \backslash\{0\},
\end{gathered}
$$

where $\frac{\tilde{p}_{n}}{\tilde{q}_{n}}$ is the continued fraction approximates to $\tilde{\alpha}$. We will denote by $W L(\gamma, \tau, \tilde{U})$ the set of such pairs and by $W L$ the union

$$
W L=\bigcup_{\gamma, \tau>0,0<\tilde{U}<\infty} W L(\gamma, \tau, \tilde{U}) .
$$

It is obvious that $W L$ is of full Lebesgue measure. Here we remark that for $\left(\tilde{\alpha}, \alpha^{\prime}\right) \in D C$, the growth of $\left\{\tilde{q}_{n}\right\}_{n \in \mathbb{N}}$ is no more than polynomial, while for $\left(\tilde{\alpha}, \alpha^{\prime}\right) \in W L$, the growth of $\left\{\tilde{q}_{n}\right\}_{n \in \mathbb{N}}$ can be super-exponential. One of our main results is the following:

Theorem 1.1. Assume that $\alpha=\left(\tilde{\alpha}, \alpha^{\prime}\right) \in W L, v \in C^{\omega}\left(\mathbb{T}^{d}, \mathbb{R}\right)$. Then there exists $\lambda_{2}=\lambda_{2}(\alpha, v)>0$ such that if $\lambda<\lambda_{2}$, then $\Sigma_{a c}(\lambda v, \alpha)$ is of positive Lebesgue measure.

For any rational independent $\alpha \in \mathbb{T}^{d}$, in order to measure how Liouvillean $\alpha$ is, one very useful definition is the following:

$$
\beta(\alpha):=\limsup _{\mathbf{k} \in \mathbb{Z}^{d}} \frac{1}{|\mathbf{k}|} \ln \frac{1}{\|\langle\mathbf{k}, \alpha\rangle\|_{\mathbb{R} / \mathbb{Z}}} .
$$

One can show that $W L \cap\left\{\alpha \in \mathbb{T}^{d} \mid \beta(\alpha)=+\infty\right\}=\varnothing$, and for any $\beta_{*} \in$ $[0,+\infty)$ we have $W L \cap\left\{\alpha \in \mathbb{T}^{d} \mid \beta(\alpha)=\beta_{*}\right\} \neq \varnothing$. Then as a direct corollary of Theorem 1.1, we have the following:

Corollary 1.1. For any $0 \leq \beta_{*}<\infty$, there exists $\alpha \in \mathbb{T}^{d}$ with $\beta(\alpha)=\beta_{*}$, and for any $v \in C^{\omega}\left(\mathbb{T}^{d}, \mathbb{R}\right)$, there exists $\lambda_{3}=\lambda_{3}(\alpha, v)>0$, such that if $\lambda<\lambda_{3}$, then $\Sigma_{a c}(\lambda v, \alpha)$ is of positive Lebesgue measure. 
1.2. Two-dimensional frequency Schrödinger operator. By Corollary 1.1, we establish the existence of ac spectrum for non-super-Liouvillean frequency, i.e., $\beta(\alpha)<\infty$, yet if the frequency is two dimensional, we are able to show more:

Theorem 1.2. There exists $\alpha \in \mathbb{T}^{2}$ satisfying $\beta(\alpha)=\infty$, and for any $v \in C^{\omega}\left(\mathbb{T}^{2}, \mathbb{R}\right)$, there exists $\lambda_{4}=\lambda_{4}(\alpha, v)>0$, such that if $\lambda<\lambda_{4}$, then $\Sigma_{a c}(\lambda v, \alpha)$ is of positive Lebesgue measure.

If the frequency is two dimensional, we can only prove the existence of ac spectrum for some super-Liouvillean frequency (i.e. $\beta(\alpha)=\infty$ ). One thus wonder whether one can prove this for all super-Liouvillean frequency, as in the one-frequency case $[3,4]$. However, this is impossible due to recent remarkable counterexample of Avila-Jitomirskaya [8], who proved that there exists $\alpha \in \mathbb{T}^{2}$ satisfying $\beta(\alpha)=\infty$, such that for typical analytic potential $v \in C^{\omega}\left(\mathbb{T}^{2}, \mathbb{R}\right), \Sigma_{a c}(v, \alpha)$ is empty. One may understand this result partially in the following way: in establishing the existence of ac spectrum for any irrational frequency, one key ingredient is the Denjoy-Koksma lemma [6]; however, this estimate fails in the multifrequency case, as pointed out by Yoccoz [32].

We also want to point out that, our result (Theorem 1.2) and AvilaJitomirskaya's [8] are both constructive. In section 3, we will show a more precise version of this result, and construct the frequency very explicitly. Readers are invited to consult Remark 3.1 for more discussions, and we will show that the existence of ac spectrum depends on quite dedicate arithmetic properties of the frequency.

\section{Preliminaries}

In this section, some frequently used facts and notations are introduced.

2.1. Notations on functions. An integrable real or complex valued function $f$ on $\mathbb{T}^{d}$ has the Fourier expansion $f=\sum_{\mathbf{k} \in \mathbb{Z}^{d}} \hat{f}(\mathbf{k}) e^{2 \pi i\langle\mathbf{k}, \phi\rangle}$ with $\hat{f}(\mathbf{k})=$ $\int_{\mathbb{T}^{d}} f(\phi) e^{-2 \pi i\langle\mathbf{k}, \phi\rangle} d \phi$. For any $N>0, \mathcal{T}_{N}$ and $\mathcal{R}_{N}$ are used to denote the truncation operators:

$$
\mathcal{T}_{N}(f)=\sum_{|\mathbf{k}|<N} \hat{f}(\mathbf{k}) e^{2 \pi i\langle\mathbf{k}, \phi\rangle}, \mathcal{R}_{N}(f)=\sum_{|\mathbf{k}| \geq N} \hat{f}(\mathbf{k}) e^{2 \pi i\langle\mathbf{k}, \phi\rangle}
$$

Let $\mathfrak{r}>0$, and we denote by $C_{\mathfrak{r}}^{\omega}\left(\mathbb{T}^{d}, \mathbb{R}\right)\left(C_{\mathfrak{r}}^{\omega}\left(\mathbb{T}^{d}, \mathbb{C}\right)\right)$ the set of all real (complex) valued functions admitting an analytic extension on

$$
\mathbb{T}_{\mathfrak{r}}^{d}:=\left\{\phi \in \mathbb{T}^{d}|| \Im \phi_{1}|\leq \mathfrak{r}, \cdots,| \Im \phi_{d} \mid \leq \mathfrak{r}\right\}
$$

For any $f \in C_{\mathfrak{r}}^{\omega}\left(\mathbb{T}^{d}, \mathbb{R}\right)\left(f \in C_{\mathfrak{r}}^{\omega}\left(\mathbb{T}^{d}, \mathbb{C}\right)\right)$, there is

$$
\|f\|_{\mathfrak{r}}:=\sup _{\phi \in \mathbb{T}_{\mathfrak{r}}^{d}}|f(\phi)|<\infty,
$$

and it is well-known that $|\hat{f}(\mathbf{k})| \leq\|f\|_{\mathfrak{r}} e^{-2 \pi|\mathbf{k}| \mathfrak{r}}$ for all $\mathbf{k} \in \mathbb{Z}^{d}$. 
In this article, we also frequently consider real (complex) valued functions admitting an analytic extension on

$$
\mathbb{T}_{r, s}^{d}:=\left\{\phi \in \mathbb{T}^{d}|| \Im \phi_{1}|\leq r,| \Im \phi_{2}|\leq s, \cdots,| \Im \phi_{d} \mid \leq s\right\}
$$

where $r, s>0$, and use $C_{r, s}^{\omega}\left(\mathbb{T}^{d}, \mathbb{R}\right)\left(C_{r, s}^{\omega}\left(\mathbb{T}^{d}, \mathbb{C}\right)\right)$ to denote the set of all such functions. Any $f$ in $C_{r, s}^{\omega}\left(\mathbb{T}^{d}, \mathbb{R}\right)\left(C_{r, s}^{\omega}\left(\mathbb{T}^{d}, \mathbb{C}\right)\right)$ satisfies

$$
\|f\|_{r, s}:=\sup _{\phi \in \mathbb{T}_{r, s}^{d}}|f(\phi)|<\infty,
$$

and $|\hat{f}(k, l)| \leq\|f\|_{r, s} e^{-2 \pi(|k| r+|l| s)}$ for all $(k, l) \in \mathbb{Z} \times \mathbb{Z}^{d-1}$.

Let $M_{2}$ denote the set of all 2 by 2 complex matrices and $\|\cdot\|$ denote the matrix norm. An integrable $M_{2}$ valued function $F$ on $\mathbb{T}^{d}$ has the Fourier expansion $F=\sum_{\mathbf{k} \in \mathbb{Z}^{d}} \hat{F}(\mathbf{k}) e^{2 \pi i\langle\mathbf{k}, \phi\rangle}$ with $\hat{F}(\mathbf{k})=\int_{\mathbb{T}^{d}} F(\phi) e^{-2 \pi i\langle\mathbf{k}, \phi\rangle} d \phi$. For any $N>0$, we define truncation operators $\mathcal{T}_{N}$ and $\mathcal{R}_{N}$ in similar way. Let $C_{\mathfrak{r}}^{\omega}\left(\mathbb{T}^{d}, M_{2}\right)$ denotes the set of all $M_{2}$-valued functions on $\mathbb{T}^{d}$ admitting an analytic extension on $\mathbb{T}_{\mathfrak{r}}^{d}$. For any $F \in C_{\mathfrak{r}}^{\omega}\left(\mathbb{T}^{d}, M_{2}\right)$ we define

$$
\|F\|_{\mathfrak{r}}:=\sup _{\phi \in \mathbb{T}_{\mathfrak{r}}^{d}}\|F(\phi)\|,
$$

and there are estimates $\|\hat{F}(\mathbf{k})\| \leq\|F\|_{\mathfrak{r}} e^{-2 \pi|\mathbf{k}| \mathfrak{r}}\left(\mathbf{k} \in \mathbb{Z}^{d}\right)$. We also use $C_{\mathfrak{r}}^{\omega}\left(\mathbb{T}^{d}, *\right)$ to denote the set of $*$-valued functions in $C_{\mathfrak{r}}^{\omega}\left(\mathbb{T}^{d}, M_{2}\right)$, where * can be $\operatorname{sl}(2, \mathbb{R}), S L(2, \mathbb{R}), P S L(2, \mathbb{R})^{2}$ etc. Similarly, for $r, s>0$, one can also define $C_{r, s}^{\omega}\left(\mathbb{T}^{d}, *\right)$ with corresponding norm $\|\cdot\|_{r, s}$ on it.

2.2. Continued fraction expansion and CD bridge. Let $\alpha \in \mathbb{R}^{1}$ be irrational. Define $a_{0}=[\alpha], \alpha_{0}=\alpha-a_{0}$, and inductively for $j \geq 1$,

$$
a_{j}=\left[\alpha_{j-1}^{-1}\right], \quad \alpha_{j}=\left\{\alpha_{j-1}^{-1}\right\}=\alpha_{j-1}^{-1}-a_{j} .
$$

We define $p_{0}=0, p_{1}=1, q_{0}=1, q_{1}=a_{1}$ and inductively,

$$
p_{j}=a_{j} p_{j-1}+p_{j-2}, \quad q_{j}=a_{j} q_{j-1}+q_{j-2} .
$$

Then the sequence $\left(q_{n}\right)$ satisfies

$$
\begin{aligned}
& \forall 1 \leq q<q_{n}, \quad\|q \alpha\|_{\mathbb{R} / \mathbb{Z}} \geq\left\|q_{n-1} \alpha\right\|_{\mathbb{R} / \mathbb{Z}}, \\
& \frac{1}{q_{n}+q_{n+1}} \leq\left\|q_{n} \alpha\right\|_{\mathbb{R} / \mathbb{Z}} \leq \frac{1}{q_{n+1}} .
\end{aligned}
$$

Let us now introduce the CD bridge.

Definition 2.1 ([6]). Let $\mathcal{A}, \mathcal{B}, \mathcal{C} \in \mathbb{R}$ with $0<\mathcal{A} \leq \mathcal{B} \leq \mathcal{C}$. We say that the pair of denominators $\left(q_{l}, q_{n}\right)$ forms a $\operatorname{CD}(\mathcal{A}, \mathcal{B}, \mathcal{C})$ bridge if

- $q_{j+1} \leq q_{j}^{\mathcal{A}}, \quad \forall j=l, \ldots, n-1$

- $q_{l}^{\mathcal{C}} \geq q_{n} \geq q_{l}^{\mathcal{B}}$.

In the following, for simplicity, we will fix a subsequence $\left(q_{n_{j}}\right)$ of $\left(q_{n}\right)$, denoted by $\left(Q_{j}\right)$, and the subsequence $\left(q_{n_{j}+1}\right)$, denoted by $\left(\bar{Q}_{j}\right)$.

\footnotetext{
${ }^{2} \operatorname{PSL}(2, \mathbb{R})$ denotes the quotient group $S L(2, \mathbb{R}) /\{ \pm I\}$.
} 
Lemma $2.1([6])$. For any $\mathcal{A}>0$, there exists a subsequence $\left(Q_{j}\right)$ such that $Q_{0}=1$ and for each $j \geq 0, Q_{j+1} \leq \bar{Q}_{j}^{\mathcal{A}^{4}}$. Furthermore, either $\bar{Q}_{j} \geq Q_{j}^{\mathcal{A}}$, or the pairs $\left(\bar{Q}_{j-1}, Q_{j}\right)$ and $\left(Q_{j}, Q_{j+1}\right)$ are both $\operatorname{CD}\left(\mathcal{A}, \mathcal{A}, \mathcal{A}^{3}\right)$ bridges.

Corollary 2.1 ([28]). Let $\alpha \in \mathbb{R} \backslash \mathbb{Q}$. If $\widetilde{U}(\alpha)<\infty$, then for the subsequence $\left(Q_{j}\right)$ selected in Lemma 2.1 we have $Q_{j} \geq Q_{j-1}^{\mathcal{A}}$ for every $j \geq 1$. Furthermore, we have

$$
\sup _{j>0} \frac{\ln \ln Q_{j+1}}{\ln Q_{j}} \leq U(\alpha)
$$

where $U(\alpha):=\widetilde{U}(\alpha)+4 \frac{\ln \mathcal{A}}{\ln 2}<\infty$.

2.3. Cocycles, Fibered rotation number. Let $\alpha \in \mathbb{T}^{d}$ be rational independent and $G=S L(2, \mathbb{R}), S O(2, \mathbb{R})$, etc.. A quasi-periodic $G$ cocycle is a pair $(\alpha, A) \in \mathbb{T}^{d} \times C^{r}\left(\mathbb{T}^{d}, G\right)(r=0,1, \cdots, \infty, \omega)$, which represents

$$
(\alpha, A): \mathbb{T}^{d} \times \mathbb{R}^{2} \rightarrow \mathbb{T}^{d} \times \mathbb{R}^{2},(\phi, x) \mapsto(\phi+\alpha, A(\phi) x) .
$$

We consider the iterations of $(\alpha, A)$ which can be written as

$$
(\alpha, A)^{\circ n}=\underbrace{(\alpha, A) \circ \cdots \circ(\alpha, A)}_{n \text { times }}=\left(n \alpha, A^{(n)}\right)
$$

where

$$
A^{(n)}(\cdot)= \begin{cases}A(\cdot+(n-1) \alpha) \cdots A(\cdot), & \text { as } n>0, \\ I, & \text { as } n=0, \\ A(\cdot+n \alpha)^{-1} \cdots A(\cdot-\alpha)^{-1}, & \text { as } n<0 .\end{cases}
$$

We say that two cocycles $(\alpha, A),(\alpha, \widetilde{A})$ are $C^{r}$ conjugate, if there exists $B \in C^{r}\left(\mathbb{T}^{d}, P S L(2, \mathbb{R})\right)$, such that

$$
\widetilde{A}(\cdot)=B(\cdot+\alpha) A(\cdot) B^{-1}(\cdot) .
$$

Furthermore, if $\widetilde{A} \in C^{r}\left(\mathbb{T}^{d}, S O(2, \mathbb{R})\right)$ we say that $(\alpha, A)$ is $C^{r}$ rotations reducible. It is obvious that rotations reducible cocycles are always bounded.

For any $C^{0}$ quasi-periodic $S L(2, \mathbb{R})$ cocycle $(\alpha, A)$ with $A$ being homotopic to identity, it induces a projective cocycle on $\mathbb{T}^{d} \times \mathbb{S}^{1}$

$$
f_{(\alpha, A)}: \mathbb{T}^{d} \times \mathbb{S}^{1} \rightarrow \mathbb{T}^{d} \times \mathbb{S}^{1},(\phi, \vec{e}(x)) \mapsto\left(\phi+\alpha, \frac{A(\phi) \vec{e}(x)}{\|A(\phi) \vec{e}(x)\|}\right),
$$

with $\vec{e}(x)=(\cos 2 \pi x, \sin 2 \pi x)^{T}$, which is also homotopic to identity. Then $f_{(\alpha, A)}$ has a lift $F_{(\alpha, A)}: \mathbb{T}^{d} \times \mathbb{R} \circlearrowleft$ of the form $(\phi, x) \mapsto\left(\phi+\alpha, \tilde{f}_{A}(\phi, x)\right)$ with $\tilde{f}_{A}(\phi, x)=x+d_{A}(\phi, x)$, where $d_{A}$ is $\mathbb{Z}$-periodic in both variables, such that $\vec{e}\left(\tilde{f}_{A}(\phi, x)\right)=\frac{A(\phi) \vec{e}(x)}{\|A(\phi) \vec{e}(x)\|}$. As $\alpha$ has rationally independent coordinates, then the limit

$$
\tilde{\rho}(\alpha, A):=\lim _{n \rightarrow \infty} \frac{\tilde{f}_{A}\left(F_{(\alpha, A)}^{n-1}(\phi, x)\right)-x}{n}
$$


exists for all $(\phi, x) \in \mathbb{T}^{d} \times \mathbb{R}$ and it is independent of $(\phi, x)[23]$. We call $\rho(\alpha, A):=\tilde{\rho}(\alpha, A)(\bmod 1)$ the fibered rotation number of $(\alpha, A)$.

One can establish fibered rotation numbers for Schrödinger cocyles $\left(\alpha, S_{E-v}\right)$, since $S_{E-v}$ is obviously homotopic to identity, where $E \in \mathbb{R}$ and $S_{E-v}(\cdot)=$ $\left(\begin{array}{cc}E-v(\cdot) & -1 \\ 1 & 0\end{array}\right)$. As for $\left(\alpha, S_{E-v}\right)$, we always have $\rho\left(\alpha, S_{E-v}\right) \in\left[0, \frac{1}{2}\right]$.

Let $\mu_{\alpha, v, \phi}$ be the spectral measure of $H_{v, \alpha, \phi}$, and the integrated density of states $N_{\alpha, v}: \mathbb{R} \rightarrow[0,1]$ is defined as

$$
N_{\alpha, v}(E)=\int_{\mathbb{T}^{d}} \mu_{\alpha, v, \phi}(-\infty, E] d \phi .
$$

There is a close relation between $\rho\left(\alpha, S_{E-v}\right)$ and $N_{\alpha, v}(E)$ [7]:

$$
N_{\alpha, v}(E)=1-2 \rho\left(\alpha, S_{E-v}\right) .
$$

For any $g \in C^{0}\left(\mathbb{T}^{d}, \mathbb{R}\right)$, denote $R_{g}:=\left(\begin{array}{cc}\cos 2 \pi g & -\sin 2 \pi g \\ \sin 2 \pi g & \cos 2 \pi g\end{array}\right)=e^{-2 \pi g J}$, where $J=\left(\begin{array}{cc}0 & 1 \\ -1 & 0\end{array}\right)$. Then we have the following facts about fibered rotation number (one can refer to [1] for more details).

Lemma 2.2. Let $\alpha \in \mathbb{T}^{d}$ be rational independent and $A \in C^{0}\left(\mathbb{T}^{d}, S L(2, \mathbb{R})\right)$ be homotopic to identity. We have:

(1) For rotation $R_{\varrho}$ with $\varrho \in \mathbb{R}$, there is

$$
|\rho(\alpha, A)-\varrho| \leq\left\|A-R_{\varrho}\right\|_{0} .
$$

(2) For general $C \in S L(2, \mathbb{R})$, there exists $c=c(C) \geq 1$, such that

$$
|\rho(\alpha, A)-\rho(\alpha, C)| \leq c\|A-C\|_{0}^{1 / 2} .
$$

Usually the fibered rotation number varies after a conjugation via some $B \in C^{0}\left(\mathbb{T}^{d}, P S L(2, \mathbb{R})\right)$, but the change depends only on the topological degree of $B$. We say that the topological degree of $B$ is $\mathbf{d} \in \mathbb{Z}^{d}$, as long as $B$ is homotopic to

$$
\left(\begin{array}{cc}
\cos \pi\langle\mathbf{d}, \theta\rangle & -\sin \pi\langle\mathbf{d}, \theta\rangle \\
\sin \pi\langle\mathbf{d}, \theta\rangle & \cos \pi\langle\mathbf{d}, \theta\rangle
\end{array}\right)
$$

and we use $\operatorname{deg}(B)$ to denote it.

Lemma $2.3([27])$. Let $A \in C^{0}\left(\mathbb{T}^{d}, S L(2, \mathbb{R})\right)$ and it is homotopic to identity, $B \in C^{0}\left(\mathbb{T}^{d}, P S L(2, \mathbb{R})\right)$. If $\widetilde{A} \in C^{0}\left(\mathbb{T}^{d}, S L(2, \mathbb{R})\right)$ and $(\alpha, A)$ is conjugate to $(\alpha, \widetilde{A})$ via $B$, then $\widetilde{A}$ is also homotopic to identity and

$$
\rho(\alpha, \widetilde{A})=\rho(\alpha, A)+\frac{1}{2}\langle\operatorname{deg}(B), \alpha\rangle \quad(\bmod \quad \mathbb{Z}) .
$$

In particular, $\rho(\alpha, \widetilde{A})=\rho(\alpha, A)$ if $B$ is homotopic to identity. 


\section{Proof of MAin Results}

The proof relies on the following well-known criterion of ac spectrum, which comes from the subordinacy theory.

Proposition 3.1 ([21]). Let $\widetilde{\mathcal{O}}$ be the set of $E \in \mathbb{R}$ such that the cocycle $\left(\alpha, S_{E-v}\right)$ is bounded. If $\widetilde{\mathcal{O}}$ has positive Lebesgue measure, then $H_{v, \alpha, \phi}$ has absolutely continuous spectrum for any $\phi$.

To obtain energies $E$, such that the corresponding cocycle $\left(\alpha, S_{E-v}\right)$ is bounded, our proof is purely dynamical. Recall that rotations reducible cocycles are always bounded. Then the key dynamical results are the following:

Theorem 3.1. Let $\varrho_{0} \in \mathbb{R}, \mathfrak{r}>0, F \in C_{\mathfrak{r}}^{\omega}\left(\mathbb{T}^{d}, s l(2, \mathbb{R})\right)$. Suppose that $\alpha \in W L$, and the fibered rotation number $\rho\left(\alpha, R_{\varrho_{0}} e^{F}\right)=: \varrho \in D_{\alpha}\left(\gamma^{\prime}, \tau^{\prime}\right)$, i.e. there exist $\gamma^{\prime}>0, \tau^{\prime}>0$ such that

$$
\|2 \varrho+\langle k, \alpha\rangle\|_{\mathbb{R} / \mathbb{Z}} \geq \frac{\gamma^{\prime}}{(1+|k|)^{\tau^{\prime}}}, \quad \forall k \in \mathbb{Z}^{d} .
$$

Then there exists $\varepsilon_{*}=\varepsilon_{*}\left(\alpha, \gamma^{\prime}, \tau^{\prime}, \mathfrak{r}\right)>0$, such that if $\|F\|_{\mathfrak{r}}<\varepsilon_{*}$, then $\left(\alpha, R_{\varrho_{0}} e^{F}\right)$ is $C^{\infty}$ rotations reducible.

This result is for the multifrequency case, and if the frequency is two dimensional, then we first need to construct the super-Liouvillean frequency. The construction of the frequencies is as follows: for any two real numbers $\tilde{\alpha}, \alpha^{\prime} \in(0,1)$, suppose that $\left\{\tilde{q}_{n}\right\}_{n \in \mathbb{N}},\left\{q_{n}^{\prime}\right\}_{n \in \mathbb{N}}$ are the convergences of $\tilde{\alpha}$ and $\alpha^{\prime}$ respectively.

Lemma 3.1. For any $\chi \geq 5$, denote $\Omega(\chi) \subseteq \mathbb{T}^{2}$ the set of rational independent $\alpha=\left(\tilde{\alpha}, \alpha^{\prime}\right) \in \mathbb{T}^{2}$ satisfying the following properties:

(a) $\tilde{q}_{n}>e^{q_{n-1}^{\prime}}$;

(b) $\tilde{q}_{n}^{5}<q_{n}^{\prime}<4 \tilde{q}_{n}^{\chi}$;

(c) $\tilde{q}_{n} \wedge q_{n-1}^{\prime}=1$;

(d) $q_{n}^{\prime} \wedge \tilde{q}_{n}=1$.

Then $\Omega(\chi) \neq \varnothing$ and $\Omega(\chi) \subseteq\left\{\alpha \in \mathbb{T}^{2} \mid \beta(\alpha)=\infty\right\}$.

Proof. Let us first prove that $\Omega(\chi) \neq \varnothing$. We construct the sequences $\left\{\tilde{a}_{n}\right\}_{n \geq 1},\left\{a_{n}^{\prime}\right\}_{n \geq 1}$ of $\tilde{\alpha}, \alpha^{\prime}$ respectively by induction. For $n=1$, we let $\tilde{a}_{1}=2$ and $a_{1}^{\prime}=37$. Then $q_{1}^{\prime}$ and $\tilde{q}_{1}$ satisfy (a)-(d) for $n=1$. For $n \geq 2$, suppose that we have already chosen $\tilde{a}_{j}, a_{j}^{\prime}, j \leq n-1$, such that $(a)-(d)$ hold until the $n-1^{t h}$ step. Since $\tilde{q}_{n-1}$ and $q_{n-1}^{\prime}$ are coprime, there exist $\tilde{s}_{n}, \tilde{t}_{n} \in \mathbb{Z}$ with $\left|\tilde{s}_{n}\right|<\tilde{q}_{n-1} \tilde{q}_{n-2},\left|\tilde{t}_{n}\right|<q_{n-1}^{\prime} \tilde{q}_{n-2}$, such that

$$
\tilde{t}_{n} \tilde{q}_{n-1}+\tilde{q}_{n-2}=\tilde{s}_{n} q_{n-1}^{\prime} \text {. }
$$

Moreover, there exists $\tilde{k}_{n} \in \mathbb{Z}_{+}$, such that $\tilde{k}_{n} \wedge q_{n-1}^{\prime}=1$, and

$$
\tilde{k}_{n} \tilde{q}_{n-1} \geq 2 e^{q_{n-1}^{\prime}} \text {. }
$$


Let $\tilde{a}_{n}=\tilde{t}_{n}+\tilde{k}_{n} \in \mathbb{Z}_{+}$. Then we have

$$
\tilde{q}_{n}=\tilde{a}_{n} \tilde{q}_{n-1}+\tilde{q}_{n-2}=\tilde{k}_{n} \tilde{q}_{n-1}+\tilde{t}_{n} \tilde{q}_{n-1}+\tilde{q}_{n-2} .
$$

Thus, we obtain that $\tilde{q}_{n} \wedge q_{n-1}^{\prime}=1$, and

$$
\tilde{q}_{n} \geq 2 e^{q_{n-1}^{\prime}}-q_{n-1}^{\prime} \tilde{q}_{n-1} \tilde{q}_{n-2}>e^{q_{n-1}^{\prime}},
$$

since $q_{n-1}^{\prime} \geq q_{1}^{\prime}=37$. As for the construction of $a_{n}^{\prime}$, we follow the same idea: let $a_{n}^{\prime}=t_{n}^{\prime}+k_{n}^{\prime}$, where $t_{n}^{\prime} q_{n-1}^{\prime}+q_{n-2}^{\prime}$ divides $\tilde{q}_{n}, k_{n}^{\prime} \wedge \tilde{q}_{n}=1$, and $2 \tilde{q}_{n}^{5} \leq k_{n}^{\prime} q_{n-1}^{\prime} \leq 3 \tilde{q}_{n}^{\chi}$. Then we get $(b)$ and $(d)$ at the $n^{\text {th }}$ step. We thus complete the proof of $\Omega(\chi) \neq \varnothing$.

Moreover, for any $\alpha \in \Omega(\chi)$, by properties (a) and (b), we get for any $n \geq 1$ that $\tilde{q}_{n}>e^{\tilde{q}_{n-1}^{5}}$. Therefore, we have $\Omega(\chi) \subseteq\left\{\alpha \in \mathbb{T}^{2} \mid \beta(\alpha)=\infty\right\}$.

Remark 3.1. In Avila-Jitomirskaya's result [8], they choose the alternating super-Liouvillean frequency, i.e.

$\left(a^{\prime}\right) \quad \tilde{q}_{n}>e^{q_{n-1}^{\prime}} ;$

$\left(b^{\prime}\right) \quad q_{n}^{\prime}>e^{\tilde{q}_{n}}$.

Therefore, compared to our frequency, the main difference is (b), while we can also choose $\tilde{q}_{n}$ arbitrarily larger than $q_{n-1}^{\prime}$, we must assume $q_{n}^{\prime}$ grows polynomially with respect to $\tilde{q}_{n}$.

Once we choose the frequency, then we have the following rotations reducibility result.

Theorem 3.2. Let $\mathfrak{r}, \gamma^{\prime}, \tau^{\prime}>0, \chi \geq 5, F \in C_{\mathfrak{r}}^{\omega}\left(\mathbb{T}^{d}, \operatorname{sl}(2, \mathbb{R})\right)$. Suppose that $\alpha \in \Omega(\chi)$ and $\rho\left(\alpha, R_{\varrho_{0}} e^{F}\right) \in D_{\alpha}\left(\gamma^{\prime}, \tau^{\prime}\right)$. Then there exists $\varepsilon_{*}=$ $\varepsilon_{*}\left(\alpha, \gamma^{\prime}, \tau^{\prime}, \mathfrak{r}\right)>0$, such that if $\|F\|_{\mathfrak{r}}<\varepsilon_{*}$, then $\left(\alpha, R_{\varrho_{0}} e^{F}\right)$ is $C^{\infty}$ rotations reducible.

The proof of Theorem 3.1 and Theorem 3.2 will be left to section 4 and section 5. Now let's finish the proof of Theorem 1.1 and Theorem 1.2 based on the above results. Here we only give the proof of Theorem 1.2, and the proof of Theorem 1.1 is the same. In order to apply Proposition 3.1 to prove Theorem 1.2, we have to obtain positive Lebesgue measure of the energies $E$ such that the corresponding cocycles are bounded, while Theorem 3.2 only gives us positive Lebesgue measure of the fibered rotation numbers (not the energies) such that the corresponding cocycle is bounded. Hence, we need the following elementary result, and this kind of idea first appeared in [5].

Lemma $3.2([13$, Lemma 3.9]). Let $I, J$ be two intervals of $\mathbb{R}$ and $f: I \rightarrow J$ be a continuous map. Assume that there exists a set $\Upsilon \subseteq J$, of positive Lebesgue measure, such that $\forall x \in f^{-1}(\Upsilon)$ there is a constant $c_{x}>0$ such that $\left|f(x)-f\left(x^{\prime}\right)\right| \leq c_{x}\left|x-x^{\prime}\right|$ for all $x^{\prime}$ close to $x$. Then $f^{-1}(\Upsilon)$ has positive Lebesgue measure.

Once we have this, we can now finish the whole proof. 
Proof of Theorem 1.2. By Lemma 3.1, we can select $\alpha \in \Omega(\chi) \subseteq$ $\left\{\alpha \in \mathbb{T}^{2} \mid \beta(\alpha)=\infty\right\}$. Fix some absolutely small $0<\sigma<<1$. Note $S_{E-\lambda v}=\left(\begin{array}{cc}E & -1 \\ 1 & 0\end{array}\right) \exp \left\{\lambda v(\phi)\left(\begin{array}{cc}0 & 0 \\ 1 & 0\end{array}\right)\right\}$. One can find $E_{1}<E_{2}$, such that

$$
\left[2 \sigma, \frac{1}{2}-2 \sigma\right] \subseteq \rho_{0}\left(\left[E_{1}, E_{2}\right]\right) \subseteq\left[\sigma, \frac{1}{2}-\sigma\right]
$$

where we denote $\rho_{0}(E)=\rho\left(\alpha,\left(\begin{array}{cc}E & -1 \\ 1 & 0\end{array}\right)\right)$. Then, $\forall E \in\left[E_{1}, E_{2}\right], \exists P_{E} \in$ $S L(2, \mathbb{R})$, such that $P_{E}\left(\begin{array}{cc}E & -1 \\ 1 & 0\end{array}\right) P_{E}^{-1}=R_{\rho_{0}(E)}$. Moreover, $P_{E}$ are uniformly bounded on $\left[E_{1}, E_{2}\right]$.

Denote $\rho_{\lambda v}(E)=\rho\left(\alpha, S_{E-\lambda v}\right)$ for short. By (3.2) and Lemma 2.2, there exists $\epsilon^{\prime}>0$, when $\|\lambda v\|_{\mathfrak{r}}<\epsilon^{\prime}$, we have $\left|\rho_{\lambda v}(E)-\rho_{0}(E)\right|<\sigma$, which implies that $\rho_{\lambda v}\left(\left[E_{1}, E_{2}\right]\right) \supseteq\left[3 \sigma, \frac{1}{2}-3 \sigma\right]$ and then $\rho_{\lambda v}\left(\left[E_{1}, E_{2}\right]\right) \cap D_{\alpha}\left(\gamma^{\prime}, \tau^{\prime}\right)$ is of positive measure. Then $P_{E}$ conjugates $\left(\alpha, S_{E-\lambda v}\right)$ to $\left(\alpha, R_{\rho_{0}(E)} e^{F_{E}}\right)$, where $F_{E}(\cdot):=\lambda v(\cdot) P_{E}\left(\begin{array}{cc}0 & 0 \\ 1 & 0\end{array}\right) P_{E}^{-1}$. Let $\varepsilon_{*}>0$ be the one given in Theorem 3.2. There exists $\epsilon^{\prime \prime} \in\left(0, \epsilon^{\prime}\right]$, such that when $\|\lambda v\|_{\mathfrak{r}}<\epsilon^{\prime \prime}$ and $\rho_{\lambda v}(E) \in$ $D_{\alpha}\left(\gamma^{\prime}, \tau^{\prime}\right)$, we have $\left\|F_{E}\right\|_{\mathfrak{r}}<\varepsilon_{*}$ and $\rho\left(\alpha, R_{\rho_{0}(E)} e^{F_{E}}\right) \in D_{\alpha}\left(\gamma^{\prime}, \tau^{\prime}\right)$. Therefore, by Theorem 3.2, $\left(\alpha, R_{\rho_{0}(E)} e^{F_{E}}\right)$ is rotations reducible and so is $\left(\alpha, S_{E-\lambda v}\right)$. Let $\Pi\left(\gamma^{\prime}, \tau^{\prime}\right):=\left\{E \in\left[E_{1}, E_{2}\right] \mid \rho_{\lambda v}(E) \in D_{\alpha}\left(\gamma^{\prime}, \tau^{\prime}\right)\right\}$. When $\|\lambda v\|_{\mathfrak{r}}<\epsilon^{\prime \prime}$ and $E \in \Pi\left(\gamma^{\prime}, \tau^{\prime}\right)$, then $\left(\alpha, S_{E-\lambda v}\right)$ is rotations reducible. In order to apply Lemma 3.2, we need the following lemmas:

Lemma 3.3. Let $\alpha \in \mathbb{T}^{d}$ be rational independent and $A \in C^{0}\left(\mathbb{T}^{d}, S L(2, \mathbb{R})\right)$ be homotopic to identity. For any $S O(2, \mathbb{R})$-valued $R_{g}$ with $g \in C^{0}\left(\mathbb{T}^{d}, \mathbb{R}\right)$, there exists an absolute constant $c_{*} \geq 1$, such that if $\left\|A-R_{g}\right\|_{0}<\frac{1}{4}$, then

$$
\left|\rho(\alpha, A)-\rho\left(\alpha, R_{g}\right)\right| \leq c_{*}\left\|A-R_{g}\right\|_{0} .
$$

Proof. Denote $\tilde{A}:=R_{-g} A$, and then $A=R_{g} \tilde{A}$. Then $(\alpha, A)=\left(\alpha, R_{g}\right) \circ$ $(0, \tilde{A})$, and thus

$$
F_{(\alpha, A)}(\phi, x)=F_{\left(\alpha, R_{g}\right)} \circ F_{(0, \tilde{A})}(\phi, x)=\left(\phi+\alpha, x+g(\phi)+d_{\tilde{A}}(\phi, x)\right),
$$

where $F_{(\alpha, A)}$ is a lift of $f_{(\alpha, A)}$ as in section 2.3. Since $F_{\left(\alpha, R_{g}\right)}(\phi, x)=(\phi+$ $\alpha, x+g(\phi))$, then by the definition of fibered rotation number, we get that

$$
\left|\rho(\alpha, A)-\rho\left(\alpha, R_{g}\right)\right| \leq\left\|d_{\tilde{A}}\right\|_{0} .
$$

By a direct computation, there exists $c_{*} \geq 1$ such that for $\left\|A-R_{g}\right\|_{0}<\frac{1}{4}$ we have

$$
\left\|d_{\tilde{A}}\right\|_{0} \leq c_{*}\|\tilde{A}-I\|_{0} \leq c_{*}\left\|R_{-g}\right\|_{0}\left\|A-R_{g}\right\|_{0} \leq c_{*}\left\|A-R_{g}\right\|_{0} .
$$

Consequently, we have

$$
\left|\rho(\alpha, A)-\rho\left(\alpha, R_{g}\right)\right| \leq c_{*}\left\|A-R_{g}\right\|_{0} .
$$


Lemma 3.4. If $\left(\alpha, S_{E-v}\right)$ is rotations reducible, there exists a constant $c_{E}>$ 0 , such that $\left|\rho_{v}(E)-\rho_{v}\left(E^{\prime}\right)\right| \leq c_{E}\left|E-E^{\prime}\right|$ for all $E^{\prime}$ close to $E$.

Proof. We assume that $\left(\alpha, S_{E-v}\right)$ is conjugate via $B$ to $\left(\alpha, R_{g}\right)$. Since $\left(\alpha, S_{E-v}\right)$ is homotopic to the identity, the same is true for $\left(\alpha, R_{g}\right)$ by Lemma 2.3. Thus, we have $g \in C^{0}\left(\mathbb{T}^{2}, \mathbb{R}\right)$. Moreover, by Lemma 2.3, we obtain that $\rho_{v}(E)=\rho\left(\alpha, R_{g}\right)-\frac{1}{2}\langle\operatorname{deg}(B), \alpha\rangle(\bmod \mathbb{Z})$ and $\rho_{v}\left(E^{\prime}\right)=$ $\rho\left(\alpha, B(\cdot+\alpha) S_{E^{\prime}-v}(\cdot) B^{-1}(\cdot)\right)-\frac{1}{2}\langle\operatorname{deg}(B), \alpha\rangle(\bmod \mathbb{Z})$. Let $F(\cdot)=B(\cdot+$ $\alpha)\left(\begin{array}{ll}1 & 0 \\ 0 & 0\end{array}\right) B^{-1}(\cdot)$. Then obviously, we have $\|F\|_{0} \leq\|B\|_{0}^{2}$. For $E^{\prime}=$ $E+\Delta E$, there is

$$
B(\cdot+\alpha) S_{E^{\prime}-v}(\cdot) B^{-1}(\cdot)=R_{g}(\cdot)+\Delta E \cdot F(\cdot) .
$$

Then for $E^{\prime}$ close to $\mathrm{E}$, we have $\|\Delta E F\|_{0}<\frac{1}{4}$, and by Lemma 3.3, we get

$$
\left|\rho\left(\alpha, R_{g}\right)-\rho\left(\alpha, R_{g}+\Delta E \cdot F\right)\right| \leq c_{*}|\Delta E||| F \|_{0}<c_{E}|\Delta E|,
$$

which implies the desired conclusion.

Consequently, by Lemma 3.4 and Lemma $3.2, \Pi\left(\gamma^{\prime}, \tau^{\prime}\right)$ is of positive measure. By Proposition 3.1, we thus finish the whole proof.

\section{Proof of Theorem 3.1}

In this section, we use KAM iteration to prove Theorem 3.1. The philosophy of KAM iteration is to find a sequence of coordinate transformations through which the perturbations gradually become smaller and smaller. In the end, the transformations converge and the limit conjugates the original system to some simpler one.

However, our proof relies on a modified KAM scheme. In conjugating a cocycle $\left(\alpha, R_{\varrho} e^{F}\right)$ to some simpler one, a crucial ingredient is to solve the linearized homological equations in the form

$$
h_{1}(\cdot+\alpha)-h_{1}+f_{1}=f_{1}^{(u)}, \quad e^{4 \pi i \varrho} h_{2}(\cdot+\alpha)-h_{2}+f_{2}=0,
$$

where $h_{1}, h_{2}$ are unknown, $f_{1}, f_{2}$ comes from $F$, and $f_{1}^{(u)}$ is the unsolvable part. When $\alpha$ is Diophantine, $f_{1}^{(u)}$ is a constant and $\left(\alpha, R_{\varrho} e^{F}\right)$ is conjugated to $\left(\alpha, R_{\varrho_{+}} e^{F_{+}}\right)\left(\varrho_{+} \in \mathbb{R}\right)$ with $F_{+}$being much smaller. We then solve new linearized homological equations in similar form [19]. But, for $\alpha \in W L, f_{1}^{(u)}$ will depend on $\varphi:=\phi_{1} \in \mathbb{T}^{1}$, where $\phi=\left(\phi_{1}, \cdots, \phi_{d}\right) \in \mathbb{T}^{d}$. As a result, we arrive at $\left(\alpha, R_{\varrho+\frac{g(\varphi)}{2 \pi}} e^{F_{+}(\phi)}\right)$ with $F_{+}$being much smaller while $g$ is as large as $F$. Thus, in our KAM iteration, we should consider linearized homological equation in the form

$$
e^{2 i(2 \pi \varrho+g(\varphi))} h(\cdot+\alpha)-h+f=0,
$$

with unknown $h$. It is the essential difference from the classical one [19] and we deduce it using techniques in [28]. 
In the sequel, for $\phi=\left(\phi_{1}, \cdots, \phi_{d}\right) \in \mathbb{T}^{d}$, we denote $\varphi:=\phi_{1} \in \mathbb{T}^{1}, \theta:=$ $\left(\phi_{2}, \cdots, \phi_{d}\right) \in \mathbb{T}^{d-1}$ for simplicity.

4.1. Linearized Homological Equation. To prove Theorem 3.1, one needs to consider the linearized homological equation of the form

$$
e^{2 i(2 \pi \varrho+g(\varphi))} h(\phi+\alpha)-h(\phi)=-f(\phi)
$$

with $f \in C_{r, s}^{\omega}\left(\mathbb{T}^{1} \times \mathbb{T}^{d-1}, \mathbb{C}\right)$ and $g \in C_{r}^{\omega}\left(\mathbb{T}^{1}, \mathbb{R}\right)$ for some $r, s>0$. Such an equation can be furthermore written in the form

$$
\left(e^{4 \pi i \varrho}+\widetilde{g}(\varphi)\right) h(\phi+\alpha)-h(\phi)=-f(\phi)
$$

where $\widetilde{g} \in C_{r}^{\omega}\left(\mathbb{T}^{1}, \mathbb{R}\right)$ defined as

$$
\widetilde{g}(\varphi)=e^{4 \pi i \varrho}\left(e^{2 i g(\varphi)}-1\right) .
$$

Solving such kind of linearized homological equations will play an important role in the KAM-type proof of Theorem 3.1. We solve (4.2) by an approximate equation, which is the content of the following proposition.

Proposition 4.1. Let $\gamma^{\prime}>0, \tau^{\prime}>0, r>\sigma>0, s>\delta>0, \sigma \leq \delta \leq \frac{1}{4}$, $0<\tilde{\eta} \leq \eta \ll 1$, and $g \in C_{r}^{\omega}\left(\mathbb{T}^{1}, \mathbb{R}\right), f \in C_{r, s}^{\omega}\left(\mathbb{T}^{1} \times \mathbb{T}^{d-1}, \mathbb{C}\right)$. If $\|g\|_{r} \leq \eta$, $\|f\|_{r, s} \leq \tilde{\eta}$

$$
K=\left[\frac{1}{2 \pi \sigma} \ln \frac{1}{\tilde{\eta}}\right]+1<\left(\frac{\gamma^{\prime} \sigma}{32 \pi \eta}\right)^{\frac{1}{\tau^{\prime}}},
$$

and $\varrho \in D_{\alpha}\left(\gamma^{\prime}, \tau^{\prime}\right)$, then (4.1) has an approximate solution $h \in C_{r-\sigma, s-\delta}^{\omega}\left(\mathbb{T}^{1} \times\right.$ $\left.\mathbb{T}^{d-1}, \mathbb{C}\right)$ with

$$
\|h\|_{r-\sigma, s-\delta} \leq \frac{C_{0}(d) K^{\tau^{\prime}}}{\gamma^{\prime} \sigma^{d}} \cdot \tilde{\eta},
$$

and the error term $\tilde{P}=\mathcal{R}_{K}(\tilde{g}(\varphi) h(\phi+\alpha)+f(\phi))$ with $\tilde{g}$ satisfying (4.3) and

$$
\|\tilde{P}\|_{r-2 \sigma, s-2 \delta} \leq C_{1}(d) K^{d}\left(1+\frac{K^{\tau^{\prime}}}{\gamma^{\prime} \sigma^{d}} \eta\right) \cdot \tilde{\eta}^{2},
$$

where $C_{0}, C_{1}$ are constants only depending on $d$.

The proof of Proposition 4.1 is somehow technical and we put it in the Appendix.

4.2. KAM Step. In this section, we will give the inductive lemma and details about one step of the iteration.

Before stating the inductive lemma, we first give some notations. Let $\alpha:=\left(\tilde{\alpha}, \alpha^{\prime}\right)$. For any $r, s, \eta, \tilde{\eta}, \gamma^{\prime}, \tau^{\prime}>0$, we define

$$
\begin{aligned}
& \mathcal{F}_{r, s}\left(\eta, \tilde{\eta}, \gamma^{\prime}, \tau^{\prime}\right):= \\
& \left\{\begin{array}{c}
\left(\alpha, R_{\varrho+\frac{g(\varphi)}{2 \pi}} e^{F(\varphi, \theta)}\right) \in C_{r, s}^{\omega}\left(\mathbb{T}^{1} \times \mathbb{T}^{d-1}, S L(2, \mathbb{R})\right): \\
\rho\left(\alpha, R_{\varrho+\frac{g}{2 \pi}} e^{F}\right) \in D_{\alpha}\left(\gamma^{\prime}, \tau^{\prime}\right) \text { and }\|g\|_{r} \leq \eta,\|F\|_{r, s} \leq \tilde{\eta}
\end{array}\right\} .
\end{aligned}
$$


Without loss of generality, we assume that there is some $\gamma, \tau>0$ and $\tilde{U} \in[0,+\infty)$, such that $\alpha=\left(\tilde{\alpha}, \alpha^{\prime}\right) \in W L(\gamma, \tau, \tilde{U})$. Suppose that $\left(Q_{j}\right)$ is the selected sequence of $\tilde{\alpha}$ by Lemma 2.1 with $\mathcal{A}=4$ and $U=\tilde{U}(\tilde{\alpha})+8$. For $\gamma, \gamma^{\prime}>0, \tau, \tau^{\prime}>0$, let

$$
\tau_{*}=\max \left\{\tau, \tau^{\prime}\right\}, \quad \gamma_{*}=\min \left\{\gamma, \gamma^{\prime}\right\} .
$$

Let $r_{0}>0, s_{0}>0$, and $Q_{*} \in \mathbb{N}$ be the smallest integer such that for any $Q>Q_{*}$ we have

$$
\ln Q<\frac{Q^{1 / 8} r_{0}}{40 c \tau_{*} U}
$$

where $\mathrm{c}$ is a global constant with $c>100$. Now suppose $\varepsilon_{0}$ is small enough such that

$$
\varepsilon_{0}<\min \left\{\frac{\left(r_{0} s_{0} \gamma_{*} C_{*}^{-1}\right)^{60\left(\tau_{*}+d\right)}}{\left(\tau_{*}+d\right) ! Q_{1}^{12 c \tau_{*} U}}, e^{-2 c \tau_{*} U}, e^{-40\left(\ln Q_{*}\right)^{2} c \tau_{*} U}\right\}, \quad \ln \frac{1}{\varepsilon_{0}}<\left(\frac{1}{\varepsilon_{0}}\right)^{\frac{1}{12 \tau_{*}}}
$$

For any given $r_{0}, s_{0}, \varepsilon_{0}$ satisfying (4.5), let $\Delta_{0}=s_{0} / 4$, and we inductively define some sequences depending on $r_{0}, s_{0}, \Delta_{0}, \varepsilon_{0}$ for $j \geq 1$ :

$$
\begin{array}{ll}
r_{j}=\frac{r_{0}}{2 Q_{j}^{3}} & \bar{r}_{j}=\frac{r_{0}}{Q_{j}^{3}}, \\
\Delta_{j}=\Delta_{0} / 2^{j}, & s_{j}=s_{j-1}-\Delta_{j}, \\
\varepsilon_{j}=\frac{\varepsilon_{j-1}}{Q_{j+1}^{2 j+1} c_{*} U}, & \tilde{\varepsilon}_{j}=\sum_{m=0}^{j-1} \varepsilon_{m} .
\end{array}
$$

Proposition 4.2 (Iterative Lemma). For any $\varepsilon_{0}, r_{0}, s_{0}, \gamma, \gamma^{\prime}, \tau, \tau^{\prime}>0$ satisfying (4.5), and $\alpha=\left(\tilde{\alpha}, \alpha^{\prime}\right) \in W L(\gamma, \tau, \tilde{U})$ with $\tilde{U}=\tilde{U}(\tilde{\alpha})<\infty$, we define $\varepsilon_{n}, \tilde{\varepsilon}_{n}, r_{n}, s_{n}$ as in (4.6). Then the following holds for $n \geq 2$ : If the cocycle

$$
\left(\alpha, R_{\varrho+\frac{g_{n-1}(\varphi)}{2 \pi}} e^{F_{n-1}(\varphi, \theta)}\right) \in \mathcal{F}_{r_{n-1}, s_{n-1}}\left(4 \tilde{\varepsilon}_{n-1}, \varepsilon_{n-1}, \gamma^{\prime}, \tau^{\prime}\right),
$$

then it is conjugate to the cocycle

$$
\left(\alpha, R_{\varrho+\frac{g_{n}(\varphi)}{2 \pi}} e^{F_{n}(\varphi, \theta)}\right) \in \mathcal{F}_{r_{n}, s_{n}}\left(4 \tilde{\varepsilon}_{n}, \varepsilon_{n}, \gamma^{\prime}, \tau^{\prime}\right),
$$

by an analytic conjugation $\Phi_{n} \in C_{r_{n}, s_{n}}^{\omega}\left(\mathbb{T}^{1} \times \mathbb{T}^{d-1}, S L(2, \mathbb{R})\right)$ satisfying

$$
\left\|\Phi_{n}-I\right\|_{r_{n}, s_{n}} \leq 2 \varepsilon_{n-1}^{3 / 4} \text {. }
$$

We will divide the proof of Proposition 4.2 into different lemmas.

Lemma 4.1. For $n \geq 2$, any cocycle

$$
\left(\alpha, R_{\varrho+\frac{g(\varphi)}{2 \pi}} e^{F(\varphi, \theta)}\right) \in \mathcal{F}_{r_{n-1}, s_{n-1}}\left(4 \tilde{\varepsilon}_{n-1}, \varepsilon_{n-1}, \gamma^{\prime}, \tau^{\prime}\right)
$$

can be conjugate to another cocycle

$$
\left(\alpha, R_{\rho_{f}+\frac{\tilde{g}(\varphi)}{2 \pi}} e^{\tilde{F}(\varphi, \theta)}\right) \in \mathcal{F}_{\bar{r}_{n}, s_{n-1}}\left(\varepsilon_{n-1}^{3 / 4}, 2 \varepsilon_{n-1}, \gamma^{\prime}, \tau^{\prime}\right),
$$


where $\rho_{f}=\rho\left(\alpha, R_{\varrho+\frac{g}{2 \pi}} e^{F}\right)$ via some conjugation $R_{\frac{v_{n}(\varphi)}{2 \pi}}=e^{-v_{n}(\varphi) J}$, with $v_{n}$ satisfying

$$
\left\|v_{n}\right\|_{\bar{r}_{n}}<Q_{n}^{\frac{7}{4}} \varepsilon_{0}^{1 / 2}
$$

Moreover, the fibered rotation number remains unchanged.

Proof. Let $v_{n}$ be the solution of

$$
v_{n}(\varphi+\tilde{\alpha})-v_{n}(\varphi)=-\mathcal{T}_{Q_{n}} g(\varphi)+\hat{g}(0) .
$$

Then we have

$$
\left\|v_{n}\right\|_{\frac{r_{n-1}}{2}} \leq 2 Q_{n} \sum_{0<|k|<Q_{n}}\|g\|_{r_{n-1}} e^{-2 \pi|k| r_{n-1} / 2} \leq \frac{64 Q_{n} \varepsilon_{0}}{r_{n-1}} \leq Q_{n}^{\frac{7}{4}} \varepsilon_{0}^{1 / 2},
$$

and the given cocycle is conjugate to $\left(\alpha, R_{\tilde{\varrho}+\frac{\mathcal{R}_{Q_{n} g(\varphi)}}{2 \pi}} e^{\tilde{F}(\varphi, \theta)}\right)$, where

$$
\tilde{\varrho}=\varrho+\frac{\hat{g}(0)}{2 \pi}, \quad \tilde{F}(\varphi, \theta)=e^{-v_{n}(\varphi) J} F(\varphi, \theta) e^{v_{n}(\varphi) J} .
$$

Here we need a small trick used in $[28,33]$, saying $\left|\Im v_{n}(\varphi)\right|$ can be well controlled by sacrificing the analytic radius. We give a short review of the proof for completeness.

Lemma 4.2. Let $r>0, \tilde{\omega} \in \mathbb{R}^{m}(m \in \mathbb{N})$, and $v(\varphi)$ is the solution of $v(\varphi+\tilde{\omega})-v(\varphi)=\mathcal{T}_{K} g(\varphi)-\hat{g}(0)$, where $g \in C_{r}^{\omega}\left(\mathbb{T}^{m}, \mathbb{R}\right)$ and $K \in \mathbb{N}$. If $\|\langle k, \tilde{\omega}\rangle\|_{\mathbb{R} / \mathbb{Z}} \geq \iota^{-1}>0$ for all $0<|k|<K\left(k \in \mathbb{Z}^{m}\right)$, then for any $\bar{r}<r$, we have

$$
\|\Im v\|_{\bar{r}} \leq \frac{C \bar{r} \iota\|g\|_{r}}{(r-\bar{r})^{m+1}}
$$

where $C$ is a global constant depending on $m$.

Proof. Let $\varphi=\varphi_{1}+i \varphi_{2}$, with $\varphi_{1} \in \mathbb{T}^{m}, \varphi_{2} \in \mathbb{R}^{m}$. Denote

$$
v_{1}(\varphi)=v\left(\varphi_{1}\right), \text { and } v_{2}(\varphi)=v(\varphi)-v_{1}(\varphi)
$$

Owing to the fact that $g(\varphi)$ is real-analytic in $\varphi$, we get $v_{1}(\varphi) \in \mathbb{R}$. Therefore,

$$
\begin{aligned}
& \|\Im v\|_{\bar{r}}=\left\|\Im v_{2}\right\|_{\bar{r}} \\
& \quad \leq \sup _{\left|\varphi_{2}\right|<\bar{r}} \sum_{0<|k|<K} \iota|\hat{g}(k)| \cdot\left|e^{-2 \pi\left\langle k, \varphi_{2}\right\rangle}-1\right| \\
& \leq \quad \iota \sum_{0<|k|<K}\|g\|_{r} e^{-2 \pi|k|(r-\bar{r})} \cdot 2 \pi|k| \bar{r} \leq \frac{C \bar{r} \iota\|g\|_{r}}{(r-\bar{r})^{m+1}} .
\end{aligned}
$$

By Lemma 4.2, we get (as $\varepsilon_{0}$ is sufficiently small)

$$
\left\|\Im v_{n}\right\|_{\bar{r}_{n}} \leq \frac{C r_{0}^{-1} \tilde{\varepsilon}_{n-1}}{Q_{n}^{1 / 2}} \ll 1
$$


Thus, we have

$$
\|\tilde{F}\|_{\bar{r}_{n}, s_{n-1}}<2 \varepsilon_{n-1} .
$$

Moreover, standard computation shows that for $\varepsilon_{0}$ small enough we have

$$
\left\|\mathcal{R}_{Q_{n}} g\right\|_{r_{n-1} / 2} \leq C Q_{n} e^{-2 \pi Q_{n} r_{n-1} / 2}\|g\|_{r_{n-1}} \leq \varepsilon_{n-1}^{4 / 5},
$$

by (4.5) and the selection of $Q_{*}$. Since $e^{v_{n}(\varphi) J}$ is homotopic to the identity, by Lemma 2.2 and 2.3, the fibered rotation number of the new system remains $\rho_{f}$, and $\left|\rho_{f}-\tilde{\varrho}\right| \leq 2\left(\left\|\mathcal{R}_{Q_{n}} g\right\|_{0}+\|\tilde{F}\|_{0}\right) \leq 4 \varepsilon_{n-1}^{4 / 5}$. Let $\tilde{g}(\varphi)=\mathcal{R}_{Q_{n}} g(\varphi)+$ $2 \pi\left(\tilde{\varrho}-\rho_{f}\right)$. Then

$$
\|\tilde{g}\|_{\bar{r}_{n}} \leq\left\|\mathcal{R}_{Q_{n}} g\right\|_{r_{n-1} / 2}+2 \pi\left|\rho_{f}-\tilde{\varrho}\right|<\varepsilon_{n-1}^{3 / 4} .
$$

We finish the proof of Lemma 4.1 .

Remark 4.1. In the proof of Lemma 4.1, although the norm of the transformation $e^{v_{n} J}$ is not large, it may not be close to the identity.

Once we get the cocycle (4.10), we will use Proposition 4.1 to make the perturbation small enough.

Lemma 4.3. Under the assumptions of Lemma 4.1, for $n \geq 1$, there exists a conjugation map $\Psi_{n} \in C_{r_{n}, s_{n}}^{\omega}\left(\mathbb{T}^{1} \times \mathbb{T}^{d-1}, S L(2, \mathbb{R})\right)$ with

$$
\left\|\Psi_{n}-I\right\|_{r_{n}, s_{n}} \leq \varepsilon_{n-1}^{3 / 4},
$$

such that $\Psi_{n}$ conjugates (4.10) to

$$
\left(\alpha, R_{\rho_{f}+\frac{\tilde{g}_{+}(\varphi)}{2 \pi}} e^{\tilde{F}_{+}(\varphi, \theta)}\right) \in \mathcal{F}_{r_{n}, s_{n}}\left(2 \varepsilon_{n-1}^{3 / 4}, \varepsilon_{n} / 2, \gamma^{\prime}, \tau^{\prime}\right),
$$

satisfying

$$
\left\|\tilde{g}_{+}-\tilde{g}\right\|_{r_{n}} \leq 4 \varepsilon_{n-1}
$$

Proof. For simplicity, we denote temporarily $\tilde{r}_{0}:=\left\{\begin{array}{ll}r_{0}, & n=1 \\ \bar{r}_{n}=\frac{r_{0}}{Q_{n}^{3},} & n \geq 2\end{array}\right.$, $\tilde{s}_{0}:=s_{n-1}, \tilde{\Delta}:=\Delta_{n}, \eta:=2 \varepsilon_{n-1}^{3 / 4}, \tilde{\eta}_{0}:=2 \varepsilon_{n-1}, \tilde{g}_{0}(\varphi):=\tilde{g}(\varphi), \tilde{F}_{0}(\varphi, \theta):=$ $\tilde{F}(\varphi, \theta)$, and define sequences inductively for $\nu \geq 1:^{3}$

$$
\begin{array}{ll}
\sigma_{1}=\frac{\tilde{r_{0}}}{8}, & \sigma_{\nu}=\frac{1}{2^{\nu-1}} \sigma_{1}, \\
\delta_{1}=\frac{\tilde{\Delta}}{4}, & \delta_{\nu}=\frac{1}{2^{\nu-1}} \delta_{1}, \\
\widetilde{r}_{\nu}=\widetilde{r}_{\nu-1}-2 \sigma_{\nu}, & \widetilde{s}_{\nu}=\widetilde{s}_{\nu-1}-2 \delta_{\nu}, \\
\tilde{\eta}_{\nu}=\tilde{\eta}_{0}^{\left(\frac{3}{2}\right)^{\nu}}, & K_{\nu}=\left[\frac{1}{2 \pi \sigma_{\nu}} \ln \frac{1}{\tilde{\eta}_{\nu-1}}\right]+1 .
\end{array}
$$

Let $N=\left[2^{n} c_{1} \tau_{*} U \ln Q_{n}\right]+1$, where $c_{1}=\frac{c}{24 \tau_{*} \ln 3}$. For convenience, we denote

$$
\tilde{A}_{j}=R_{\rho_{f}+\frac{\tilde{g}_{j}}{2 \pi}} .
$$

\footnotetext{
${ }^{3}$ Note that $\frac{1}{Q_{n}^{3}}$ goes to 0 much faster than $\Delta_{n}$, we can assume $\tilde{r}_{0}<2 \tilde{\Delta}$ in general, and thus $\sigma_{\nu}<\delta_{\nu}$.
} 
Assume that for $j=1,2, \cdots, \nu-1<N(\nu \geq 1)$, there exist $Y_{j}, \tilde{F}_{j} \in$ $C_{\tilde{r}_{j}, \tilde{s}_{j}}^{\omega}\left(\mathbb{T}^{1} \times \mathbb{T}^{d-1}, s l(2, \mathbb{R})\right)$, with $\left\|Y_{j}\right\|_{\tilde{r}_{j}, \tilde{s}_{j}} \leq \tilde{\eta}_{j-1}^{4 / 5},\left\|\tilde{F}_{j}\right\|_{\tilde{r}_{j}, \tilde{s}_{j}} \leq \tilde{\eta}_{j}$, so that $e^{Y_{j}}$ conjugates the cocycle $\left(\alpha, \tilde{A}_{j-1} e^{\tilde{F}_{j-1}(\varphi, \theta)}\right)$ to the cocycle $\left(\alpha, \tilde{A}_{j} e^{\tilde{F}_{j}(\varphi, \theta)}\right)$, where $\tilde{F}_{j}=\left(\begin{array}{cc}\tilde{F}_{j}^{11} & \tilde{F}_{j}^{12} \\ \tilde{F}_{j}^{21} & -\tilde{F}_{j}^{11}\end{array}\right)$ and $\tilde{g}_{j}=\tilde{g}_{j-1}+\frac{\left[\tilde{F}_{j-1}^{12}-\tilde{F}_{j-1}^{21}\right]_{\theta}}{2}$.

The linearized homological equation in $\nu$ - 1-th step is

$$
\tilde{A}_{\nu-1}^{-1} Y_{\nu}(\cdot+\alpha) \tilde{A}_{\nu-1}-Y_{\nu}+\tilde{F}_{\nu-1} \equiv\left[\tilde{F}_{\nu-1}^{-}\right]_{\theta} J .
$$

Let $M=\frac{1}{1+i}\left(\begin{array}{cc}1 & -i \\ 1 & i\end{array}\right) \in U(2)$. Then $\tilde{F}_{\nu-1}$ can be uniquely rewritten as

$$
\tilde{F}_{\nu-1}=\left(\begin{array}{cc}
\tilde{F}_{\nu-1}^{11} & \tilde{F}_{\nu-1}^{+}+\tilde{F}_{\nu-1}^{-} \\
\tilde{F}_{\nu-1}^{+}-\tilde{F}_{\nu-1}^{-} & -\tilde{F}_{\nu-1}^{11}
\end{array}\right)=M^{-1}\left(\begin{array}{cc}
i \tilde{F}_{\nu-1}^{-} & w_{\nu-1}^{1} \\
w_{\nu-1}^{2} & -i \tilde{F}_{\nu-1}^{-}
\end{array}\right) M,
$$

where $\tilde{F}_{\nu-1}^{ \pm}=\frac{1}{2}\left(\tilde{F}_{\nu-1}^{12} \pm \tilde{F}_{\nu-1}^{21}\right), w_{\nu-1}^{1}=\tilde{F}_{\nu-1}^{11}-i \tilde{F}_{\nu-1}^{+}, w_{\nu-1}^{2}=\tilde{F}_{\nu-1}^{11}+i \tilde{F}_{\nu-1}^{+}$. Similarly, we can rewrite

$$
Y_{\nu}=M^{-1}\left(\begin{array}{cc}
i Y_{\nu}^{-} & h_{\nu}^{1} \\
h_{\nu}^{2} & -i Y_{\nu}^{-}
\end{array}\right) M
$$

where $Y_{\nu}^{ \pm}=\frac{1}{2}\left(Y_{\nu}^{12} \pm Y_{\nu}^{21}\right), h_{\nu}^{1}=Y_{\nu}^{11}-i Y_{\nu}^{+}, h_{\nu}^{2}=Y_{\nu}^{11}+i Y_{\nu}^{+}$. Then the homological equation (4.14) is equivalent to

$$
\begin{gathered}
Y_{\nu}^{-}(\cdot+\alpha)-Y_{\nu}^{-}=-\tilde{F}_{\nu-1}^{-}+\left[\tilde{F}_{\nu-1}^{-}\right]_{\theta}, \\
e^{2 i\left(2 \pi \rho_{f}+\tilde{g}_{\nu-1}\right)} h_{\nu}^{1}(\cdot+\alpha)-h_{\nu}^{1}=-w_{\nu-1}^{1}, \\
e^{-2 i\left(2 \pi \rho_{f}+\tilde{g}_{\nu-1}\right)} h_{\nu}^{2}(\cdot+\alpha)-h_{\nu}^{2}=-w_{\nu-1}^{2} .
\end{gathered}
$$

Since $\left(\tilde{\alpha}, \alpha^{\prime}\right) \in W L(\gamma, \tau, \tilde{U})$, it is classical to solve (4.15) with

$$
\left\|Y_{\nu}^{-}\right\|_{\tilde{r}_{\nu}, \tilde{s}_{\nu}} \leq \frac{4(\tau+d) ! \tilde{\eta}_{\nu-1}}{\gamma \sigma_{\nu}^{\tau+d}}<\tilde{\eta}_{\nu-1}^{4 / 5}
$$

We will apply Proposition 4.1 to solve (4.16). First, we can check that

$$
\left\|\tilde{g}_{\nu-1}\right\|_{\tilde{r}_{\nu-1}} \leq\left\|\tilde{g}_{0}\right\|_{\tilde{r}_{0}}+\sum_{j=0}^{\nu-2} \tilde{\eta}_{j}<2 \varepsilon_{n-1}^{3 / 4}=\eta .
$$

Moreover, for $\nu \leq N$, we have

$$
\begin{aligned}
K_{\nu} & \leq K_{N} \leq \frac{1}{2 \pi \sigma_{N}} \ln \frac{1}{\tilde{\eta}_{N-1}}+1=\frac{4 \cdot 3^{N-1} Q_{n}^{3}}{\pi r_{0}} \ln \frac{1}{2 \varepsilon_{n-1}}+1 \\
& <\frac{2 Q_{n}^{\frac{2^{n} c U}{24}}+3}{r_{0}}\left(\frac{1}{\varepsilon_{n-1}}\right)^{\frac{1}{12 \tau_{*}}}<\left(\frac{\gamma^{\prime} \sigma_{N}}{32 \pi \cdot 2 \varepsilon_{n-1}^{3 / 4}}\right)^{\frac{1}{\tau^{\prime}}} .
\end{aligned}
$$

Then by the assumption $\rho_{f} \in D_{\alpha}\left(\gamma^{\prime}, \tau^{\prime}\right)$, we can apply Proposition 4.1 to (4.16), getting an approximate solution $h_{\nu}^{1}$, with the error term $\tilde{P}_{\nu}^{1}$, satisfying 
the estimations

$$
\begin{aligned}
& \left\|h_{\nu}^{1}\right\|_{\tilde{r}_{\nu-1}-\sigma_{\nu}, \tilde{s}_{\nu-1}-\delta_{\nu}} \leq \frac{C_{0}(d) K_{\nu}^{\tau^{\prime}} \tilde{\eta}_{\nu-1}}{\gamma^{\prime} \sigma_{\nu}^{d}}<\tilde{\eta}_{\nu-1}^{4 / 5}, \\
& \left\|\tilde{P}_{\nu}^{1}\right\|_{\tilde{r}_{\nu}, \tilde{s}_{\nu}}<\frac{C_{1}(d) K_{\nu}^{d} \tilde{\eta}_{\nu-1}^{2}}{\sigma_{\nu}^{d-1}}<\tilde{\eta}_{\nu-1}^{7 / 4} .
\end{aligned}
$$

Denote

$$
h_{\nu}^{\iota}(\varphi, \theta)=\sum_{k \in \mathbb{Z}, l \in \mathbb{Z}^{d-1}} \hat{h}_{\nu, l}^{\iota}(k) e^{2 \pi i k \varphi+2 \pi i\langle l, \theta\rangle}, \quad \iota=1,2 .
$$

If we let $\hat{h}_{\nu, l}^{2}(k)=\overline{\hat{h}_{\nu,-l}^{1}(-k)}$, then by the relation between $w_{\nu-1}^{1}$ and $w_{\nu-1}^{2}$, as well as (4.16) and (4.17), we obtain that $h_{\nu}^{2}$ is an approximate solution of (4.17), with the error term $\tilde{P}_{\nu}^{2}$, satisfying the same estimates as in (4.18). Moreover, it holds that $h_{\nu}^{1}+h_{\nu}^{2}, i\left(h_{\nu}^{1}-h_{\nu}^{2}\right), \tilde{P}_{\nu}^{1}+\tilde{P}_{\nu}^{2}, i\left(\tilde{P}_{\nu}^{1}-\tilde{P}_{\nu}^{2}\right) \in C_{\tilde{r}_{\nu}, \tilde{s}_{\nu}}^{\omega}\left(\mathbb{T}^{1} \times\right.$ $\left.\mathbb{T}^{d-1}, \mathbb{R}\right)$. Then we have

$$
\begin{aligned}
& A_{\nu-1}^{-1} Y_{\nu}(\cdot+\alpha) A_{\nu-1}-Y_{\nu}+\tilde{F}_{\nu-1} \\
= & \left(\begin{array}{cc}
0 & {\left[\tilde{F}_{\nu-1}^{-}\right]_{\theta}} \\
-\left[\tilde{F}_{\nu-1}^{-}\right]_{\theta} & 0
\end{array}\right)+\left(\begin{array}{cc}
\frac{1}{2}\left(\tilde{P}_{\nu}^{1}+\tilde{P}_{\nu}^{2}\right) & \frac{i}{2}\left(\tilde{P}_{\nu}^{1}-\tilde{P}_{\nu}^{2}\right) \\
\frac{i}{2}\left(\tilde{P}_{\nu}^{1}-\tilde{P}_{\nu}^{2}\right) & -\frac{1}{2}\left(\tilde{P}_{\nu}^{1}+\tilde{P}_{\nu}^{2}\right)
\end{array}\right) \\
= & :\left(\begin{array}{cc}
0 & {\left[\tilde{F}_{\nu-1}^{-}\right]_{\theta}} \\
-\left[\tilde{F}_{\nu-1}^{-}\right]_{\theta} & 0
\end{array}\right)+\tilde{P}_{\nu .}
\end{aligned}
$$

In conclusion, we obtain that $Y_{\nu}, \tilde{P}_{\nu} \in C_{\tilde{r}_{\nu}, \tilde{s}_{\nu}}^{\omega}\left(\mathbb{T}^{1} \times \mathbb{T}^{d-1}, s l(2, \mathbb{R})\right)$, with

$$
\left\|Y_{\nu}\right\|_{\tilde{r}_{\nu-1}-\sigma_{\nu}, \tilde{s}_{\nu-1}-\delta_{\nu}}<\tilde{\eta}_{\nu-1}^{4 / 5}, \quad\left\|\tilde{P}_{\nu}\right\|_{\tilde{r}_{\nu}, \tilde{s}_{\nu}}<\tilde{\eta}_{\nu-1}^{7 / 4}
$$

Let

$$
\tilde{A}_{\nu}=\tilde{A}_{\nu-1} e^{\left[\tilde{F}_{\nu-1}^{-}\right]_{\theta} J}, \text { and } E_{\nu}=\tilde{A}_{\nu}^{-1} e^{Y_{\nu}(\cdot+\alpha)} \tilde{A}_{\nu-1} e^{F_{\nu-1}} e^{-Y_{\nu}} .
$$

Then by standard estimation (c.f. [19] for example), we have

$$
\begin{aligned}
\left\|E_{\nu}-i d\right\|_{\tilde{r}_{\nu}, \tilde{s}_{\nu}} & \leq C\left(\left\|\tilde{F}_{\nu-1}\right\|^{2}+\left\|Y_{\nu}\right\|^{2}+\left\|\tilde{F}_{\nu-1}\right\| \cdot\left\|Y_{\nu}\right\|+\left\|\tilde{P}_{\nu}\right\|\right) \\
& \leq C \tilde{\eta}_{\nu-1}^{8 / 5} \ll 1 .
\end{aligned}
$$

Thus, by the implicit function theorem, there exists $\tilde{F}_{\nu} \in C_{\tilde{r}_{\nu}, \tilde{s}_{\nu}}^{\omega}\left(\mathbb{T}^{1} \times\right.$ $\left.\mathbb{T}^{d-1}, \operatorname{sl}(2, \mathbb{R})\right)$ such that $E_{\nu}=e^{\tilde{F}_{\nu}}$ with $\left\|\tilde{F}_{\nu}\right\|_{\tilde{r}_{\nu}, \tilde{s}_{\nu}} \leq\left\|E_{\nu}-i d\right\|_{\tilde{r}_{\nu}, \tilde{s}_{\nu}}<\tilde{\eta}_{\nu-1}^{3 / 2}$, which completes the $\nu$-th step.

To finish the proof, we need to give the estimate of $\tilde{F}_{N}$. By the selection of $U$, we have $Q_{n}^{U} \geq \ln Q_{n+1}$. Therefore,

$$
\begin{aligned}
& \left\|\tilde{F}_{N}\right\|_{\tilde{r}_{N}, \tilde{s}_{N}} \leq \tilde{\eta}_{0}^{\left(\frac{3}{2}\right)^{N}}=\tilde{\eta}_{0} e^{-\left(\left(\frac{3}{2}\right)^{N}-1\right) \ln \frac{1}{\tilde{\eta}_{0}}}
\end{aligned}
$$

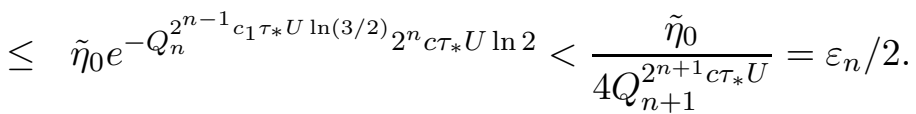

Furthermore, it is obvious that $\tilde{s}_{N}>s_{n}, \tilde{r}_{N}>r_{n}$. Let

$$
y_{+}:=y_{N}, \quad \tilde{g}_{+}:=\tilde{g}_{N}, \quad \tilde{F}_{+}:=\tilde{F}_{N} .
$$


Then we get the system (4.13) with $\left\|\tilde{g}_{+}-\tilde{g}\right\|_{r_{n}}<2 \tilde{\eta}_{0}$. Now, we give the estimate of the conjugation map $\Psi_{n}:=e^{Y_{N}} e^{Y_{N-1}} \cdots e^{Y_{1}}$, satisfying

$\left\|\Psi_{n}-I\right\|_{r_{n}, s_{n}} \leq \sum_{j=1}^{N}\left\|\left(e^{Y_{j}}-I\right) e^{Y_{j-1}} \cdots e^{Y_{2}} e^{Y_{1}}\right\|_{\tilde{r}_{j}, \tilde{s}_{j}} \leq \sum_{j=1}^{N} 4\left\|Y_{j}\right\|_{\tilde{r}_{j}, \tilde{s}_{j}}<\varepsilon_{n-1}^{3 / 4}$.

Moreover, by Lemma 2.3, since $\Psi_{n}$ is close to the identity, meaning that $\Psi_{n}$ is homotopic to the identity, we have the fibered rotation number of (4.13) is same as (4.10) and also (4.9).

As mentioned in Remark 4.1, the transformation $e^{v_{n} J}$ may not be close to the identity, which will be an obstruction on the convergence of the transformations. Therefore, we will do one more operation, which guarantees the convergence of the transformations.

Lemma 4.4. Under the assumptions of Lemma 4.3, the cocycle (4.13) can be conjugate to

$$
\left(\alpha, R_{\varrho+\frac{g_{+}(\varphi)}{2 \pi}} e^{F_{+}(\varphi, \theta)}\right) \in \mathcal{F}_{r_{n}, s_{n}}\left(4 \tilde{\varepsilon}_{n}, \varepsilon_{n}, \gamma^{\prime}, \tau^{\prime}\right)
$$

by the conjugation $R_{-\frac{v_{n}(\varphi)}{2 \pi}}$, where $v_{n}(\varphi)$ is the same as in Lemma 4.1 .

Proof. By simple computations, we know that $e^{v_{n}(\varphi) J}$ conjugates the cocycle (4.13) to $\left(\alpha, R_{\rho_{f}+\frac{\tilde{g}_{+}+\mathcal{T}_{Q_{n} g-\hat{g}(0)}}{2 \pi}} e^{v_{n} J} e^{\tilde{F}_{+}} e^{-v_{n} J}\right)$. Let

$$
g_{+}(\varphi)=2 \pi\left(\rho_{f}-\varrho\right)+\tilde{g}_{+}+\mathcal{T}_{Q_{n}} g-\hat{g}(0), \quad F_{+}(\varphi, \theta)=e^{v_{n} J} \tilde{F}_{+} e^{-v_{n} J} .
$$

Recalling that

$$
v_{n}(\varphi+\tilde{\alpha})-v_{n}(\varphi)=-\mathcal{T}_{Q_{n}} g(\varphi)+\hat{g}(0),
$$

we thus have

$g_{+}-g=2 \pi\left(\rho_{f}-\varrho\right)+\tilde{g}_{+}+\mathcal{T}_{Q_{n}} g-\hat{g}(0)-g=\tilde{g}_{+}-\tilde{g}+\mathcal{R}_{Q_{n}} g+\mathcal{T}_{Q_{n}} g-g$, which implies that $\|\tilde{g}-g\|_{r_{n}} \leq 4 \varepsilon_{n-1}$ by Lemma 4.3. And Lemma 4.1 implies

$$
\left\|F_{+}\right\|_{r_{n}, s_{n}} \leq e^{\left\|\Im v_{n}\right\|_{r_{n}}}\left\|\tilde{F}_{+}\right\|_{r_{n}, s_{n}} e^{\left\|\Im v_{n}\right\|_{r_{n}}}<\varepsilon_{n} .
$$

Moreover, by the same reason of Lemma 4.1, the fibered rotation number of (4.19) does not change.

Now we are on the position to prove Proposition 4.2.

Proof of Proposition 4.2. Let $\Phi_{n}=e^{v_{n} J} \Psi_{n} e^{-v_{n} J}$. Combining Lemma 4.1, 4.3 , and 4.4 , we only need to estimate $\Phi_{n}-I$ :

$$
\begin{aligned}
& \left\|\Phi_{n}-I\right\|_{r_{n}, s_{n}}=\left\|e^{v_{n} J} \Psi_{n} e^{-v_{n} J}-I\right\|_{r_{n}, s_{n}} \\
& \quad=\left\|e^{-v_{n} J}\left(\Psi_{n}-I\right) e^{v_{n} J}\right\|_{r_{n}, s_{n}} \\
& \quad \leq 2\left\|\Psi_{n}-I\right\|_{r_{n}, s_{n}}<2 \varepsilon_{n-1}^{3 / 4}
\end{aligned}
$$


4.3. Proof of Theorem 3.1. Let $\varepsilon_{0}=\|F\|_{\mathfrak{r}}$ satisfy (4.5), where $r_{0}=$ $s_{0}=\mathfrak{r}$, and $\left(Q_{n}\right)$ is the selected sequence of $\tilde{\alpha}$ in Lemma 2.1 with $\mathcal{A}=4$, $\tilde{U}=\tilde{U}(\tilde{\alpha})$ and $U:=\tilde{U}+8$. Define $r_{n}, s_{n}, \varepsilon_{n}, \tilde{\varepsilon}_{n}$ for $n \geq 1$ as in (4.6). Let $\rho_{f}:=\rho\left(\alpha, R_{\varrho_{0}} e^{F}\right), g_{0}:=2 \pi\left(\varrho_{0}-\rho_{f}\right)$ and $F_{0}:=F$. By the assumption, we have $\left(\alpha, R_{\rho_{f}+\frac{g_{0}}{2 \pi}} e^{F_{0}}\right) \in \mathcal{F}_{r_{0}, s_{0}}\left(8 \varepsilon_{0}, \varepsilon_{0}, \gamma^{\prime}, \tau^{\prime}\right)$. Then by Lemma 4.3 , there exists $\Psi_{1} \in C_{r_{1}, s_{1}}^{\omega}\left(\mathbb{T}^{1} \times \mathbb{T}^{d-1}, S L(2, \mathbb{R})\right)$ that conjugates $\left(\alpha, R_{\rho_{f}+\frac{g_{0}}{2 \pi}} e^{F_{0}}\right)$ to $\left(\alpha, R_{\rho_{f}+\frac{g_{1}}{2 \pi}} e^{F_{1}}\right)$ which belongs to $\mathcal{F}_{r_{1}, s_{1}}\left(4 \tilde{\varepsilon}_{1}, \varepsilon_{1}, \gamma^{\prime}, \tau^{\prime}\right)$. Let $\Phi_{1}:=\Psi_{1}$. Applying Proposition 4.2 inductively for $n \geq 2$, then we obtain a sequence of transformations $\left\{\Phi_{n}\right\}_{n \geq 2}$ such that $\Phi_{n}$ conjugates (4.7) into (4.8) with $\varrho=\rho_{f}$. Let

$$
\Phi^{(n)}=\Phi_{n} \Phi_{n-1} \cdots \Phi_{1} .
$$

Then by the estimates in Proposition 4.2, we get

$$
\begin{aligned}
\left\|\Phi^{(n+1)}-\Phi^{(n)}\right\|_{r_{n+1}, s_{n+1}} & =\left\|\left(\Phi_{n+1}-I\right) \Phi^{(n)}\right\|_{r_{n+1}, s_{n+1}} \\
& \leq 2 \varepsilon_{n}^{3 / 4} \prod_{j=1}^{n-1}\left(1+2 \varepsilon_{j}^{3 / 4}\right)<4 \varepsilon_{n}^{3 / 4} .
\end{aligned}
$$

Then $\Phi^{(n)}$ and $g_{n}$ converge to $\Phi, g_{\infty}$ respectively in $C^{0}$ topology. Hence, $\Phi$ conjugates $\left(\alpha, R_{\varrho_{0}} e^{F}\right)$ to the cocycle $\left(\alpha, R_{\rho_{f}+\frac{g_{\infty}(\varphi)}{2 \pi}}\right)$. Now, we certificate that $\Phi(\cdot)$ is actually in $C^{\infty}$, that is $\left\{\Phi^{(n)}(\cdot)\right\}_{n \in \mathbb{N}}$ converges in $C^{\infty}$ topology. By the definition of $\left\{\varepsilon_{n}\right\}_{n \in \mathbb{N}}$, for any $j \in \mathbb{N}^{d}$, there exists $n_{j} \in \mathbb{N}$ such that for any $n \geq n_{j}$, we have $Q_{n}^{-1}<r_{0} / 2$ and $Q_{n}^{4|j|}<\varepsilon_{n-1}^{-1 / 2}$, which implies

$$
\left(2 r_{0}^{-1} Q_{n}^{3}\right)^{|j|} \varepsilon_{n-1}^{3 / 4}<\varepsilon_{n-1}^{1 / 4}, \forall n \geq n_{j} .
$$

Then for any $j \in \mathbb{N}^{d}, n \geq n_{j}$, by Cauchy estimates, we have

$$
\left|D^{j}\left(\Phi^{(n+1)}-\Phi^{(n)}\right)\right| \leq \frac{\left\|\Phi^{(n+1)}-\Phi^{(n)}\right\|_{r_{n+1}, s_{n+1}}}{r_{n+1}^{|j|}} \leq\left(\frac{2 Q_{n+1}^{3}}{r_{0}}\right)^{|j|} \cdot 4 \varepsilon_{n}^{3 / 4}<4 \varepsilon_{n}^{1 / 4} .
$$

Hence, the limit $\Phi$ is in $C^{\infty}$, which also means $g_{\infty} \in C^{\infty}\left(\mathbb{T}^{1}, \mathbb{R}\right)$.

\section{Proof of Theorem 3.2}

In this section, we also use KAM iterations to prove Theorem 3.2. Similar as in the proof of Theorem 3.1, the key point in KAM theory is to solve the linearized homological equation, which involves the small divisor problems. Therefore, we first give some important small divisor lemmas in our proof, then perform the KAM scheme, and finally verify the convergence of the iterations.

5.1. Small divisor lemmas. Let $\alpha=\left(\tilde{\alpha}, \alpha^{\prime}\right) \in \mathbb{T}^{1} \times \mathbb{T}^{1}$. In the sequel, we will fix some $\chi \geq 5$ and let $\left(\tilde{\alpha}, \alpha^{\prime}\right) \in \Omega(\chi)$. Assume that $\frac{\tilde{p}_{n}}{\tilde{q}_{n}}$ and $\frac{p_{n}^{\prime}}{q_{n}^{\prime}}$ are the continued fraction approximates to $\tilde{\alpha}$ and $\alpha^{\prime}$ respectively.

The following two small divisor lemmas are important in our proof. 
Lemma 5.1. Let $\chi \geq 5$. If $\left(\tilde{\alpha}, \alpha^{\prime}\right) \in \Omega(\chi)$, then for $0<|k|+|l|<\tilde{q}_{n}$ $(n \geq 1)$, we have

$$
\left\|k \tilde{\alpha}+l \alpha^{\prime}\right\|_{\mathbb{R} / \mathbb{Z}} \geq \frac{1}{2 q_{n}^{\prime}} .
$$

Proof. We first deal with the case $k l=0$. If $l=0$, then for $0<|k|<\tilde{q}_{n}$, we have

$$
\|k \tilde{\alpha}\|_{\mathbb{R} / \mathbb{Z}} \geq \frac{1}{2 \tilde{q}_{n}}>\frac{1}{2 q_{n}^{\prime}} .
$$

If $k=0$, then for $0<|l|<\tilde{q}_{n}<q_{n}^{\prime}$, we have

$$
\left\|l \alpha^{\prime}\right\|_{\mathbb{R} / \mathbb{Z}} \geq \frac{1}{2 q_{n}^{\prime}} .
$$

Now we suppose $k l \neq 0$. Since $\tilde{q}_{n} \wedge q_{n-1}^{\prime}=1, \tilde{q}_{n} \wedge \tilde{p}_{n}=1,|k|<\tilde{q}_{n}$, then $\tilde{q}_{n} \nmid k \tilde{p}_{n} q_{n-1}^{\prime}$, which implies that

$$
\left\|l \frac{p_{n-1}^{\prime}}{q_{n-1}^{\prime}}+k \frac{\tilde{p}_{n}}{\tilde{q}_{n}}\right\|_{\mathbb{R} / \mathbb{Z}} \geq \frac{1}{q_{n-1}^{\prime} \tilde{q}_{n}} .
$$

Moreover,

$$
\begin{gathered}
\left|l\left(\alpha^{\prime}-\frac{p_{n-1}^{\prime}}{q_{n-1}^{\prime}}\right)\right|<\frac{\tilde{q}_{n}}{q_{n-1}^{\prime} q_{n}^{\prime}}<\frac{1}{4 q_{n-1}^{\prime} \tilde{q}_{n}}, \\
\left|k\left(\tilde{\alpha}-\frac{\tilde{p}_{n}}{\tilde{q}_{n}}\right)\right|<\frac{1}{\tilde{q}_{n+1}}<\frac{1}{4 q_{n-1}^{\prime} \tilde{q}_{n}} .
\end{gathered}
$$

Therefore, we have

$$
\left\|k \tilde{\alpha}+l \alpha^{\prime}\right\|_{\mathbb{R} / \mathbb{Z}} \geq \frac{1}{2 q_{n-1}^{\prime} \tilde{q}_{n}}>\frac{1}{2 q_{n}^{\prime}} .
$$

One of the main observation for $\left(\tilde{\alpha}, \alpha^{\prime}\right) \in \Omega(\chi)$ is that if $\varrho \in D_{\alpha}(\gamma, \tau)$ for some $\gamma>0, \tau>2$, then the small divisors $\|\langle k, \alpha\rangle \pm 2 \varrho\|_{\mathbb{R} / \mathbb{Z}}$ have some uniform lower bound for $|k|$ not too large, which is the content of the following lemma:

Lemma 5.2. Let $0<\gamma<1, \tau>2, \alpha=\left(\tilde{\alpha}, \alpha^{\prime}\right) \in \Omega(\chi)$, and $\varrho \in D_{\alpha}(\gamma, \tau)$. Then for any $k \in \mathbb{Z}^{2},|k| \leq \tilde{q}_{n+1}^{1 / 2}$, we have

$$
\|\langle k, \alpha\rangle \pm 2 \varrho\|_{\mathbb{R} / Z} \geq \frac{c \gamma^{\tau+1}}{q_{n}^{\prime \tau^{2}}}
$$

where $c$ is a constant only depending on $\tau$.

Proof. Since $\|\langle k, \alpha\rangle-2 \varrho\|_{\mathbb{R} / \mathbb{Z}}=\|\langle-k, \alpha\rangle+2 \varrho\|_{\mathbb{R} / \mathbb{Z}}$, we only need to prove

$$
\|\langle k, \alpha\rangle+2 \varrho\|_{\mathbb{R} / \mathbb{Z}} \geq \frac{c \gamma^{\tau+1}}{q_{n}^{\prime \tau^{2}}} .
$$

Let $L_{n}=\frac{12 \cdot\left(2 q_{n}^{\prime}\right)^{\tau}}{\gamma}$. We divide the proof into two cases:

Case 1: $\tilde{q}_{n+1} \leq L_{n}^{2}$. Then for $|k| \leq \tilde{q}_{n+1}^{1 / 2} \leq L_{n}$, (5.1) follows directly from the Diophantine condition that $\varrho \in D_{\alpha}(\gamma, \tau)$. 
Case 2: $\tilde{q}_{n+1}>L_{n}^{2}$. Assume that

$$
\|\langle k, \alpha\rangle+2 \varrho\|_{\mathbb{R} / \mathbb{Z}}=\left|\langle k, \alpha\rangle+2 \varrho+j_{k}\right|<1
$$

for some $j_{k} \in \mathbb{Z}$. Then we have $\left|j_{k}\right|<1+|\langle k, \alpha\rangle|+2|\varrho| \leq 3+|k|$. Let $k^{\prime}=\left(j_{k}, k\right) \in \mathbb{Z}^{3}, \omega=(1, \alpha)$. Then

$$
\left|k^{\prime}\right|=\left|j_{k}\right|+|k|<3+2|k| \leq 3 \tilde{q}_{n+1}^{1 / 2}<\frac{3 \tilde{q}_{n+1}}{L_{n}},
$$

because $\tilde{q}_{n+1}>L_{n}^{2}>144 \cdot q_{n}^{\prime 2 \tau}$. There exist $l, m \in \mathbb{Z}, \tilde{k} \in \mathbb{Z}^{3}$, such that

$$
k^{\prime}=l\left(-\tilde{p}_{n}, \tilde{q}_{n}, 0\right)+m\left(-p_{n}^{\prime}, 0, q_{n}^{\prime}\right)+\left(\tilde{k}_{1}, \tilde{k}_{2}, \tilde{k}_{3}\right),
$$

with $\left|\tilde{k}_{2}\right|<\tilde{q}_{n},\left|\tilde{k}_{3}\right|<q_{n}^{\prime},|l| \leq \frac{3 \tilde{q}_{n+1}}{L_{n} \tilde{q}_{n}},|m| \leq \frac{3 \tilde{q}_{n+1}}{L_{n} q_{n}^{\prime}}$. Then,

$$
\begin{aligned}
& \|\langle k, \alpha\rangle+2 \varrho\|_{\mathbb{R} / \mathbb{Z}}=\left|\left\langle k^{\prime}, \omega\right\rangle+2 \varrho\right| \\
& \quad=\left|l\left(\tilde{\alpha} \tilde{q}_{n}-\tilde{p}_{n}\right)+m\left(\alpha^{\prime} q_{n}^{\prime}-p_{n}^{\prime}\right)+\tilde{k}_{1}+\tilde{k}_{2} \tilde{\alpha}+\tilde{k}_{3} \alpha^{\prime}+2 \varrho\right| .
\end{aligned}
$$

Since $\left|\tilde{k}_{2}\right|+\left|\tilde{k}_{3}\right|<2 q_{n}^{\prime}$, together with the fact that $\varrho \in D_{\alpha}(\gamma, \tau)$, we get

$$
\|\langle\tilde{k}, \omega\rangle+2 \varrho\|_{\mathbb{R} / \mathbb{Z}}=\left\|\tilde{k}_{2} \tilde{\alpha}+\tilde{k}_{3} \alpha^{\prime}+2 \varrho\right\|_{\mathbb{R} / \mathbb{Z}} \geq \frac{\gamma}{\left(\left|\tilde{k}_{2}\right|+\left|\tilde{k}_{3}\right|+1\right)^{\tau}} \geq \frac{\gamma}{\left(2 q_{n}^{\prime}\right)^{\tau}} .
$$

Therefore, we have

$$
\begin{aligned}
& \|\langle k, \alpha\rangle+2 \varrho\|_{\mathbb{R} / \mathbb{Z}}=\left|\langle\tilde{k}, \omega\rangle+2 \varrho+l\left(\tilde{\alpha} \tilde{q}_{n}-\tilde{p}_{n}\right)+m\left(\alpha^{\prime} q_{n}^{\prime}-p_{n}^{\prime}\right)\right| \\
& \quad \geq \| \tilde{k}, \omega\rangle+2 \varrho \|_{\mathbb{R} / \mathbb{Z}}-\left|l\left(\tilde{\alpha} \tilde{q}_{n}-\tilde{p}_{n}\right)\right|-\left|m\left(\alpha^{\prime} q_{n}^{\prime}-p_{n}^{\prime}\right)\right| \\
& \quad \geq \frac{\gamma}{\left(2 q_{n}^{\prime}\right)^{\tau}}-\frac{3 \tilde{q}_{n+1}}{L_{n} \tilde{q}_{n}} \cdot \frac{1}{\tilde{q}_{n+1}}-\frac{3 \tilde{q}_{n+1}}{L_{n} q_{n}^{\prime}} \cdot \frac{1}{q_{n+1}^{\prime}} \geq \frac{\gamma}{2\left(2 q_{n}^{\prime}\right)^{\tau}} .
\end{aligned}
$$

This completes the proof.

5.2. KAM scheme. For $\mathfrak{r}, \eta, \tilde{\eta}, \gamma>0, \tau>2$, we let

$$
\begin{aligned}
& \tilde{\mathcal{F}}_{\mathfrak{r}}(\eta, \tilde{\eta}, \gamma, \tau):= \\
& \quad\left\{\begin{array}{l}
\left(\alpha, R_{\varrho+\frac{g}{2 \pi}} e^{F}\right): g \in C_{\mathfrak{r}}^{\omega}\left(\mathbb{T}^{2}, \mathbb{R}\right), F \in C_{\mathfrak{r}}^{\omega}\left(\mathbb{T}^{2}, s l(2, \mathbb{R})\right), \\
\rho\left(\alpha, R_{\varrho+\frac{g}{2 \pi}}\right) \in D_{\alpha}(\gamma, \tau), \text { and }\|g\|_{\mathfrak{r}} \leq \eta,\|F\|_{\mathfrak{r}} \leq \tilde{\eta}
\end{array}\right\} .
\end{aligned}
$$

Without loss of generality, we assume $\mathfrak{r} \leq 1$. For given $\chi \geq 5,0<\mathfrak{r}_{0}:=$ $\mathfrak{r} \leq 1, \tau>2$, we let $\ell_{*} \geq 2$ be the smallest real number such that for any $\ell \geq \ell_{*}$, we have

$$
2^{11} \ell^{16 \chi \tau^{2}} \leq e^{\ell^{1 / 10} \mathfrak{r}_{0}}
$$

Let $\gamma>0$ be sufficiently small. Suppose $\varepsilon_{0}$ is small enough such that

$$
\varepsilon_{0}<\min \left\{c \gamma^{8(\tau+1)} \mathfrak{r}_{0}^{16}, \ell_{*}^{-16 \chi \tau^{2}}\right\},
$$

where $c>0$ is a sufficiently small constant. Let $n_{*} \in \mathbb{N}$ be the smallest natural number such that for $n>n_{*}$

$$
2^{11} \tilde{q}_{n}^{16 \chi \tau^{2}} \leq e^{\tilde{q}_{n}^{\frac{1}{10}} \mathfrak{r}_{0}} \text {, and } e^{-\tilde{q}_{n}^{\frac{1}{10}} \mathfrak{r}_{0}} \leq \varepsilon_{0} .
$$


Let $\overline{\mathfrak{r}}_{0}=\mathfrak{r}_{0}$. Now we inductively define the sequences for $n \geq 1$ :

$$
\begin{aligned}
& \mathfrak{r}_{n}=\frac{\mathfrak{r}_{0}}{2 q_{n_{*}+n-1}^{2}}, \quad \overline{\mathfrak{r}}_{n}=\frac{4 \mathfrak{r}_{0}}{q_{n_{*}+n}^{\prime 2}}, \\
& \varepsilon_{n}=\varepsilon_{n-1} e^{-\tilde{q}_{n *+n}^{1}} \mathfrak{r}_{0}^{10}, \quad \tilde{\varepsilon}_{n}=4 \sum_{j=0}^{n-1} \varepsilon_{j}^{3 / 4} .
\end{aligned}
$$

Then by induction, we have

$$
e^{-\tilde{q}_{n *+n+1}^{\frac{1}{10}} \mathfrak{r}_{0}} \leq \varepsilon_{n}
$$

Proposition 5.1 (Iterative Lemma). Let $\varepsilon_{0}, \mathfrak{r}_{0}, \gamma>0, \tau>2, \chi \geq 5$ satisfy (5.3), and $\alpha=\left(\tilde{\alpha}, \alpha^{\prime}\right) \in \Omega(\chi)$. The following holds for $n \geq 1$ : If the cocycle

$$
\left(\alpha, R_{\varrho+\frac{g_{n}}{2 \pi}} e^{F_{n}}\right) \in \tilde{\mathcal{F}}_{\mathfrak{r}_{n}}\left(\tilde{\varepsilon}_{n}, \varepsilon_{n}, \gamma, \tau\right)
$$

satisfies $\mathcal{R}_{\tilde{q}_{n_{*}+n}} g_{n}=0$, then there exists $\Phi_{n} \in C_{\mathfrak{r}_{n+1}}^{\omega}\left(\mathbb{T}^{2}, S L(2, \mathbb{R})\right)$ with $\left\|\Phi_{n}-I\right\|_{\mathfrak{r}_{n+1}}<\varepsilon_{n}^{1 / 3}$ such that $x_{n+1}=\Phi_{n} x_{n}$ conjugates the cocycle (5.7) to the cocycle

$$
\left(\alpha, R_{\varrho+\frac{g_{n+1}}{2 \pi}} e^{F_{n+1}}\right) \in \tilde{\mathcal{F}}_{\mathfrak{r}_{n+1}}\left(\tilde{\varepsilon}_{n+1}, \varepsilon_{n+1}, \gamma, \tau\right)
$$

with $\left\|g_{n+1}-g_{n}\right\|_{\mathfrak{r}_{n+1}} \leq 4 \varepsilon_{n}^{3 / 4},\left\|F_{n+1}\right\|_{\mathfrak{r}_{n+1}} \leq \varepsilon_{n+1}$, and $\mathcal{R}_{\tilde{q}_{n_{*}+n+1}} g_{n+1}=0$.

We divide the proof into three parts that corresponds to Lemma 5.3, 5.4 and 5.6, and we omit the subscript for convenience in case there is no confusing.

Lemma 5.3. For $n \geq 1$, if $\left(\alpha, R_{\varrho+\frac{g}{2 \pi}} e^{F}\right) \in \tilde{\mathcal{F}}_{\mathfrak{r}_{n}}\left(\tilde{\varepsilon}_{n}, \varepsilon_{n}, \gamma, \tau\right)$ with $\mathcal{R}_{\tilde{q}_{n_{*}+n}} g=$ 0 , then it can be conjugate to $\left(\alpha, R_{\rho_{f}} e^{\tilde{F}}\right) \in \tilde{\mathcal{F}}_{\overline{\mathfrak{r}}_{n}}\left(0,60 \varepsilon_{n}, \gamma, \tau\right)$, with $\rho_{f}=$ $\rho\left(\alpha, R_{\varrho+\frac{g}{2 \pi}} e^{F}\right)$.

Proof. Let $v$ be the solution of

$$
v(\phi+\alpha)-v(\phi)=-g(\phi)+\hat{g}(0)
$$

Then $e^{-v(\phi) J}$ conjugates $\left(\alpha, R_{\varrho+\frac{g}{2 \pi}} e^{F}\right)$ to

$$
\left(\alpha, R_{\varrho+\frac{\hat{g}(0)}{2 \pi}} e^{e^{-v J} F(\phi) e^{v J}}\right) .
$$

Applying Lemma 4.2, we get

$$
\|\Im v\|_{\overline{\mathfrak{r}}_{n}} \leq \frac{C q_{n_{*}+n-1}^{6} \varepsilon_{0}^{3 / 4}}{\mathfrak{r}_{0}^{2} q_{n_{*}+n}^{\prime}}<\varepsilon_{0}^{1 / 2} \ll 1,
$$

because $\|\langle k, \alpha\rangle\|_{\mathbb{R} / \mathbb{Z}} \geq \frac{1}{2 q_{n_{*}+n}^{\prime}}$ for $0<|k|<\tilde{q}_{n_{*}+n}$ by Lemma 5.1. Then we have

$$
\left\|e^{-v J} F e^{v J}\right\|_{\overline{\mathfrak{r}}_{n}} \leq\left\|e^{v J}\right\|_{\overline{\mathfrak{r}}_{n}}\|F\|_{\overline{\mathfrak{r}}_{n}}\left\|e^{-v J}\right\|_{\overline{\mathfrak{r}}_{n}} \leq e^{2\|I \widetilde{I} v\|_{\mathfrak{r}_{n}}}\|F\|_{\mathfrak{r}_{n}}<2 \varepsilon_{n} .
$$


By Lemma 2.2, we have

$$
\left|\varrho+\frac{\hat{g}(0)}{2 \pi}-\rho_{f}\right| \leq\left\|e^{e^{-v J} F e^{v J}}-I\right\|_{0} \leq 2\left\|e^{-v J} F e^{v J}\right\|_{0}<4 \varepsilon_{n} .
$$

Let $E=R_{\varrho+\frac{\hat{g}(0)}{2 \pi}-\rho_{f}} e^{e^{-v J} F e^{v J}}$. Then

$$
\begin{aligned}
& \|E-I\|_{\overline{\mathfrak{r}}_{n}} \leq\left\|e^{\left(2 \pi\left(\varrho-\rho_{f}\right)+\hat{g}(0)\right) J}-I\right\|+\left\|e^{e^{-v J} F e^{v J}}-I\right\|_{\overline{\mathfrak{r}}_{n}} \\
& \quad \leq 4 \pi\left|\varrho+\frac{\hat{g}(0)}{2 \pi}-\rho_{f}\right|+2\left\|e^{-v J} F e^{v J}\right\|_{\overline{\mathfrak{r}}_{n}}<60 \varepsilon_{n} .
\end{aligned}
$$

By the implicit function theorem, there exists $\tilde{F} \in C_{\overline{\mathfrak{r}}_{n}}^{\omega}\left(\mathbb{T}^{2}, s l(2, \mathbb{R})\right)$ such that $E=e^{\tilde{F}}$ with $\|\tilde{F}\|_{\overline{\mathfrak{r}}_{n}} \leq\|E-I\|_{\overline{\mathfrak{r}}_{n}}<60 \varepsilon_{n}$. Moreover, since the conjugation map $R_{\frac{v}{2 \pi}}$ is homotpoic to the identity, the fibered rotation number of the new cocycle stays the same by Lemma 2.3.

Denote by $\mathcal{B}_{\mathfrak{r}}^{\omega}\left(\mathbb{T}^{2}, \operatorname{sl}(2, \mathbb{R})\right)$ the set of $F \in C^{\omega}\left(\mathbb{T}^{2}, \operatorname{sl}(2, \mathbb{R})\right)$ satisfying

$$
|F|_{\mathfrak{r}}:=\sum_{k \in \mathbb{Z}^{2}}\|\hat{F}(k)\| e^{2 \pi|k| \mathfrak{r}}<\infty .
$$

Then for any $F \in C_{\mathfrak{r}}^{\omega}\left(\mathbb{T}^{2}, s l(2, \mathbb{R})\right)$, we have $F \in \mathcal{B}_{\mathfrak{r}_{+}}$for any $0<\mathfrak{r}_{+}<\mathfrak{r}$ with the estimate

$$
|F|_{\mathfrak{r}_{+}} \leq \frac{36}{\min \left\{1,\left(\mathfrak{r}-\mathfrak{r}_{+}\right)^{2}\right\}}\|F\|_{\mathfrak{r}}
$$

Now, for $\mathfrak{r}>0, A \in S L(2, \mathbb{R}), \eta>0$, we decompose

$$
\mathcal{B}_{\mathfrak{r}}=\mathcal{B}_{\mathfrak{r}}^{(n r e)}(\eta) \oplus \mathcal{B}_{\mathfrak{r}}^{(r e)}(\eta)
$$

such that for any $Y \in \mathcal{B}_{\mathfrak{r}}^{(\text {nre })}(\eta)$,

$$
A^{-1} Y(\cdot+\alpha) A-Y \in \mathcal{B}_{\mathfrak{r}}^{(n r e)}, \quad\left|A^{-1} Y(\cdot+\alpha) A-Y\right|_{\mathfrak{r}} \geq \eta|Y|_{\mathfrak{r}} .
$$

Lemma 5.4. For $n \geq 0$, if $\left(\alpha, R_{\rho_{f}} e^{\tilde{F}}\right) \in \tilde{\mathcal{F}}_{\overline{\mathfrak{r}}_{n}}\left(0,60 \varepsilon_{n}, \gamma, \tau\right)$, then there exists a map $\Psi \in C_{\overline{\mathfrak{r}}_{n} / 2}^{\omega}\left(\mathbb{T}^{2}, S L(2, \mathbb{R})\right)$ with $\|\Psi-I\|_{\overline{\mathfrak{r}}_{n} / 2}<4 \varepsilon_{n}^{3 / 8}$, such that the cocycle $\left(\alpha, R_{\rho_{f}} e^{\tilde{F}}\right)$ can be conjugated to

$$
\left(\alpha, R_{\rho_{f}+\frac{\bar{g}(\phi)}{2 \pi}} e^{\bar{F}(\phi)}\right) \in \tilde{\mathcal{F}}_{\overline{\mathfrak{r}}_{n} / 4}\left(120 \varepsilon_{n}, \varepsilon_{n+1} / 2, \gamma, \tau\right),
$$

with $\mathcal{R}_{\tilde{q}_{n_{*}+n+1}} \bar{g}=0$, where $\rho_{f}=\rho\left(\alpha, R_{\rho_{f}} e^{\tilde{F}}\right)$.

In order to prove Lemma 5.4, we will need the following lemma

Lemma 5.5 ([15], Lemma 3.1). Let $\varepsilon \leq(4\|A\|)^{-4}$ and $\eta \geq 13\|A\|^{2} \varepsilon^{1 / 2}$. Then for any $F \in \mathcal{B}_{\mathfrak{r}}$ with $|F|_{\mathfrak{r}} \leq \varepsilon$, there exist $Y \in \mathcal{B}_{\mathfrak{r}}, F^{(r e)} \in \mathcal{B}_{\mathfrak{r}}^{(r e)}(\eta)$ such that

$$
e^{Y(\cdot+\alpha)} A e^{F} e^{-Y}=A e^{F^{(r e)}},
$$

with $|Y|_{\mathfrak{r}} \leq \varepsilon^{1 / 2}$ and $\left|F^{(r e)}\right|_{\mathfrak{r}} \leq 2 \varepsilon$. 
Proof. (Proof of Lemma 5.4).

For $n=0$ we have $|\tilde{F}|_{\mathfrak{r}_{0} / 2} \leq C \mathfrak{r}_{0}^{-2} \varepsilon_{0}<\varepsilon_{0}^{3 / 4}$, and for $n \geq 1$,

$$
|\tilde{F}|_{\mathfrak{r}_{n} / 2} \leq C \mathfrak{r}_{0}^{-2} \tilde{q}_{n_{*}+n}^{4 \chi} \varepsilon_{n} \leq \varepsilon_{n}^{3 / 4}
$$

by (5.4). Denote $\epsilon_{n}:=\varepsilon_{n}^{3 / 4}$. Assume $\Lambda$ is a subset of $\mathbb{Z}^{2}$ with $\Lambda=-\Lambda$ such that

$$
k \in \Lambda \Rightarrow\left\|\langle k, \alpha\rangle \pm 2 \rho_{f}\right\|_{\mathbb{R} / \mathbb{Z}} \geq 13 \epsilon_{n}^{1 / 2} .
$$

Define $\mathcal{B}_{\overline{\mathfrak{r}}_{n} / 2}^{1}$ as the space of all $F \in \mathcal{B}_{\overline{\mathfrak{r}}_{n} / 2}$ of the form

$$
F(\phi)=\sum_{k \in \Lambda} M^{-1}\left(\begin{array}{cc}
0 & \hat{F}^{11}(k)-i \hat{F}^{+}(k) \\
\hat{F}^{11}(k)+i \hat{F}^{+}(k) & 0
\end{array}\right) M e^{2 \pi i\langle k, \phi\rangle}
$$

and $\mathcal{B}_{\overline{\mathfrak{r}}_{n} / 2}^{2}$ as the space of all $F \in \mathcal{B}_{\overline{\mathfrak{r}}_{n} / 2}$ of the form

$$
\begin{gathered}
F(\phi)=\sum_{k \in \mathbb{Z}^{2}} M^{-1}\left(\begin{array}{cc}
i \hat{F}^{-}(k) & 0 \\
0 & -i \hat{F}^{-}(k)
\end{array}\right) M e^{2 \pi i\langle k, \phi\rangle} \\
+\sum_{k \in \mathbb{Z}^{2} \backslash \Lambda} M^{-1}\left(\begin{array}{cc}
0 & \hat{F}^{11}(k)-i \hat{F}^{+}(k) \\
\hat{F}^{11}(k)+i \hat{F}^{+}(k) & 0
\end{array}\right) M e^{2 \pi i\langle k, \phi\rangle},
\end{gathered}
$$

where $M=\frac{1}{1+i}\left(\begin{array}{cc}1 & -i \\ 1 & i\end{array}\right)$ and $\hat{F}^{ \pm}(k)=\frac{1}{2}\left(\hat{F}^{12}(k) \pm \hat{F}^{21}(k)\right)$. It is obvious that $\mathcal{B}_{\overline{\mathfrak{r}}_{n} / 2}=\mathcal{B}_{\overline{\mathfrak{r}}_{n} / 2}^{1} \oplus \mathcal{B}_{\overline{\mathfrak{r}}_{n} / 2}^{2}$. Now, we denote $A=R_{\rho_{f}}$. One can check that for $Y \in \mathcal{B}_{\overline{\mathfrak{r}}_{n} / 2}^{1}$,

$$
\left|A^{-1} Y(\cdot+\alpha) A-Y\right|_{\overline{\mathfrak{r}}_{n} / 2} \geq 13 \epsilon_{n}^{1 / 2}|Y|_{\overline{\mathfrak{r}}_{n} / 2}
$$

and thus $\mathcal{B}_{\overline{\mathfrak{r}}_{n} / 2}^{1} \subseteq \mathcal{B}_{\overline{\mathfrak{r}}_{n} / 2}^{(n r e)}\left(13 \epsilon_{n}^{1 / 2}\right)$. Then, we can apply Lemma 5.5, getting some $\tilde{Y} \in \mathcal{B}_{\overline{\mathfrak{r}}_{n}}$ and $\tilde{F}^{(r e)}$ in the form (5.14) such that $e^{\tilde{Y}}$ conjugates $\left(\alpha, A e^{\tilde{F}}\right)$ to $\left(\alpha, A e^{\tilde{F}^{(r e)}}\right)$ with $\|\tilde{Y}\|_{\overline{\mathfrak{r}}_{n} / 2} \leq \epsilon_{n}^{1 / 2}$ and $\left\|\tilde{F}^{(r e)}\right\|_{\overline{\mathfrak{r}}_{n} / 2} \leq 2 \epsilon_{n}$. Moreover, since $\rho_{f} \in D_{\alpha}(\gamma, \tau)$, then for any $|k| \leq \tilde{q}_{n_{*}+n+1}^{1 / 2}$ by Lemma 5.2 , we have

$$
\left\|\langle k, \alpha\rangle \pm 2 \rho_{f}\right\|_{\mathbb{R} / \mathbb{Z}} \geq \frac{c \gamma^{\tau+1}}{q_{n_{*}+n}^{\prime \tau^{2}}}>13 \epsilon_{n}^{1 / 2} .
$$

The last inequality obviously holds because of (5.3), (5.4) and the selection of $\ell_{*}$. It implies that $\Lambda^{c} \subseteq\left\{k \in \mathbb{Z}^{2}|| k \mid>\tilde{q}_{n_{*}+n+1}^{1 / 2}\right\}$. We now let

$$
\bar{g}(\phi)=-\sum_{|k|<\tilde{q}_{n_{*}+n+1}} \hat{\tilde{F}}^{(r e)-}(k) e^{2 \pi i\langle k, \phi\rangle}, \text { and } E=e^{\bar{g} J} e^{\tilde{F}^{(r e)}}=R_{-\frac{\bar{g}}{2 \pi}} e^{\tilde{F}^{(r e)}} .
$$


Then we have $\mathcal{T}_{q_{n_{*}+n+1}^{1 / 2}}\left(\tilde{F}^{(r e)}+\bar{g} J\right)=0$, which implies

$$
\begin{aligned}
\left\|\tilde{F}^{(r e)}+\bar{g} J\right\|_{\overline{\mathfrak{r}}_{n} / 4} & \leq C \tilde{q}_{n_{*}+n+1} e^{-\tilde{q}_{n_{*}+n+1}^{1 / 2} \overline{\mathfrak{r}}_{n} / 4}\left\|\tilde{F}^{(r e)}+\bar{g} J\right\|_{\overline{\mathfrak{r}}_{n}} \\
& <\frac{\varepsilon_{n} e^{-\tilde{q}_{n_{*}+n+1}^{1 / 2} \overline{\mathfrak{r}}_{n} / 8}}{4} \leq \varepsilon_{n+1} / 4
\end{aligned}
$$

by (5.4) and (5.6). Then,

$$
\begin{aligned}
\|E-I\|_{\frac{\overline{\mathfrak{r}}_{n}}{4}} & =\left\|e^{\bar{g} J} e^{-\bar{g} J+\tilde{F}^{(r e)}+\bar{g} J}-e^{\bar{g} J} e^{-\bar{g} J}\right\|_{\frac{\overline{\mathfrak{s}}_{n}}{4}} \\
& \leq\left\|e^{\bar{g} J}\right\|_{\frac{\overline{\mathfrak{r}}_{n}}{4}} \cdot\left\|e^{-\bar{g} J+\tilde{F}^{(r e)}+\bar{g} J}-e^{-\bar{g} J}\right\|_{\frac{\overline{\mathfrak{r}}_{n}}{4}} \\
& \leq e^{2\|\bar{g} J\|_{\frac{\mathfrak{q}_{n}}{4}}} e^{\left\|\tilde{F}^{(r e)}+\bar{g} J\right\|_{\frac{\bar{q}_{n}}{4}}^{4}}\left\|\tilde{F}^{(r e)}+\bar{g} J\right\|_{\frac{\overline{\mathfrak{r}}_{n}}{4}} \\
& \leq 2\left\|\tilde{F}^{(r e)}+\bar{g} J\right\|_{\overline{\mathfrak{r}}_{n} / 4} \leq \varepsilon_{n+1} / 2 .
\end{aligned}
$$

By the implicit function theorem, there exists $\bar{F} \in C_{\overline{\mathfrak{r}}_{n} / 2}^{\omega}\left(\mathbb{T}^{2}, \operatorname{sl}(2, \mathbb{R})\right)$ such that $E=e^{\bar{F}}$ with $\|\bar{F}\|_{\overline{\mathfrak{r}}_{n} / 4} \leq\|E-I\|_{\overline{\mathfrak{r}}_{n} / 4} \leq \varepsilon_{n+1} / 2$.

Let $\Psi=e^{\tilde{Y}}$. Then $\|\Psi-I\|_{\overline{\mathfrak{r}}_{n} / 2} \leq 2\|\tilde{Y}\|_{\overline{\mathfrak{r}}_{n} / 2} \leq 2 \varepsilon_{n}^{3 / 8}$, and $\Psi$ conjugates the cocycle $\left(\alpha, R_{\rho_{f}} e^{\tilde{F}}\right)$ to $\left(\alpha, R_{\rho_{f}+\frac{\bar{g}(\phi)}{2 \pi}} e^{\bar{F}(\phi)}\right)$, with $\|\bar{g}\|_{\overline{\mathfrak{r}}_{n} / 4} \leq 120 \varepsilon_{n}$.

To that end, since $\Psi$ is close to the identity and thus homotopic to the identity, Lemma 2.3 implies that the fibered rotation number of the new cocycle is the same as the original one.

Lemma 5.6. Under the assumptions of Lemma 5.4, the cocycle $\left(\alpha, R_{\rho_{f}+\frac{\bar{g}}{2 \pi}} e^{\bar{F}}\right)$ can be conjugated to $\left(\alpha, R_{\varrho+\frac{g_{+}}{2 \pi}} e^{F_{+}}\right) \in \tilde{\mathcal{F}}_{\mathfrak{r}_{n+1}}\left(\tilde{\varepsilon}_{n+1}, \varepsilon_{n+1}, \gamma, \tau\right)$.

Proof. With the conjugation $e^{v(\phi) J}$, where $v$ is the same as in Lemma 5.3, we get the cocycle $\left(\alpha, R_{\rho_{f}+\frac{\bar{g}+g-\hat{g}(0)}{2 \pi}} e^{v J} e^{\bar{F}} e^{-v J}\right)$. Then similar to the proof in Lemma 5.3, we let

$$
g_{+}=2 \pi\left(\rho_{f}-\varrho\right)+\bar{g}+g-\hat{g}(0), \text { and } F_{+}=e^{v J} \bar{F} e^{-v J} .
$$

By Lemma 4.2, Lemma 5.4 and estimation (5.11), we get

$$
\begin{gathered}
\left\|g_{+}-g\right\|_{\mathfrak{r}_{n+1}} \leq 2 \pi\left|\varrho+\frac{\hat{g}(0)}{2 \pi}-\rho_{f}\right|+\|\bar{g}\|_{\mathfrak{r}_{n+1}}<\varepsilon_{n}^{3 / 4}, \\
\left\|F_{+}\right\|_{\mathfrak{r}_{n+1}} \leq\left\|e^{v J}\right\|_{\mathfrak{r}_{n+1}}^{2} \cdot\|\bar{F}\|_{\mathfrak{r}_{n+1}}<\varepsilon_{n+1} .
\end{gathered}
$$

Moreover, the fibered rotation number does not change because the conjugation map $e^{v J}$ is homotopic to the identity.

Proof of Proposition 5.1: Let $\Phi_{n}=e^{v(\phi) J} \Psi e^{-v(\phi) J}$, where $v$ is defined as in (5.9) and $\Psi$ is the map in Lemma 5.4. Then combining Lemma 5.3, 5.4, 5.6 , the cocyle (5.7) is conjugate to (5.8) via the conjugation $\Phi_{n}$. Moreover,

$$
\|\Phi-I\|_{\mathfrak{r}_{n+1}}=\left\|e^{v J}(\Psi-I) e^{-v J}\right\|_{\mathfrak{r}_{n+1}} \leq 2\|\Psi-I\|_{\overline{\mathfrak{r}}_{n} / 2} \leq 8 \varepsilon_{n}^{3 / 8}<\varepsilon_{n}^{1 / 3} .
$$


5.3. Proof of Theorem 3.2. Denote $\rho_{f}:=\rho\left(\alpha, R_{\varrho_{0}} e^{F}\right)$. Since $\left|\rho_{f}-\varrho_{0}\right| \leq$ $2\|F\|_{0}$ by Lemma 2.2 , the cocycle $\left(\alpha, R_{\varrho_{0}} e^{F}\right)$ can be rewritten as $\left(\alpha, R_{\rho_{f}} e^{\tilde{F}}\right)$ for some $\tilde{F} \in C_{\mathfrak{r}}^{\omega}\left(\mathbb{T}^{2}, \operatorname{sl}(2, \mathbb{R})\right)$ with $\|\tilde{F}\|_{\mathfrak{r}} \leq 30\|F\|_{\mathfrak{r}}$. Let $\overline{\mathfrak{r}}_{0}:=\mathfrak{r}, g_{0}(\phi):=$ $0, F_{0}(\phi):=\tilde{F}(\phi), \tilde{\varepsilon}_{0}=0$, and $\varepsilon_{0}=\left\|F_{0}\right\|_{\mathfrak{r}_{0}}$ satisfy (5.3). Define $\mathfrak{r}_{n}, \overline{\mathfrak{r}}_{n}, \varepsilon_{n}, \tilde{\varepsilon}_{n}$ as in (5.5). By the assumption, cocycle $\left(\alpha, R_{\rho_{f}} e^{F_{0}}\right) \in \tilde{\mathcal{F}}_{\mathfrak{r}_{0}}\left(0, \varepsilon_{0}, \gamma, \tau\right)$ and applying Lemma 5.4 we get the cocycle $\left(\alpha, R_{\rho_{f}+\frac{\bar{g}_{0}}{2 \pi}} e^{\bar{F}_{0}}\right) \in \tilde{\mathcal{F}}_{\mathfrak{r}_{1}}\left(\tilde{\varepsilon}_{1}, \varepsilon_{1}, \gamma, \tau\right)$ by conjugation map $\Psi_{0}$. Let $g_{1}:=\bar{g}_{0}, F_{1}:=\bar{F}_{0}, \Phi_{0}:=\Psi_{0}$. Then the iterative lemma Proposition 5.1 applies. Inductively, we obtain the sequence of transformations $\left\{\Phi_{n}\right\}_{n \geq 0}$ with $\left\|\Phi_{n}-I\right\|_{\mathfrak{r}_{n+1}}<\varepsilon_{n}^{1 / 3}$ that transforms (5.7) to $(5.8)$.

Let

$$
\Phi^{(n)}=\Phi_{n-1} \cdots \Phi_{0}, \Phi=\lim _{n \rightarrow \infty} \Phi^{(n)}, g_{\infty}=\lim _{n \rightarrow \infty} g_{n}(\phi) .
$$

Then under the transformation $x_{\infty}=\Phi x$, we get the system $\left(\alpha, R_{\varrho+\frac{g_{\infty}}{2 \pi}}\right)$. Moreover, we can verify that $\Phi$ is $C^{\infty}$ in $\phi$ : First, by standard computation we get the estimate

$$
\left\|\Phi^{(n+1)}-\Phi^{(n)}\right\|_{\mathfrak{r}_{n+1}}<2 \varepsilon_{n}^{1 / 3} .
$$

Furthermore, by the selection of $(\alpha, \tilde{\alpha})$, for any $j \in \mathbb{Z}_{+}^{2}$ there exists $n_{j} \in \mathbb{N}$ such that for any $n \geq n_{j}$, we have $\left(\frac{32}{\mathfrak{r}_{0}} \tilde{q}_{n_{*}+n}^{2 \chi}\right)^{|j|}<e^{\frac{\tilde{q}_{n_{*}+n}^{1 / 10} \mathfrak{r}_{0}}{16}}<\varepsilon_{n}^{-1 / 8}$. Then for all $j \in \mathbb{Z}_{+}^{2}, n \geq n_{j}$, by Cauchy estimates we get

$$
\left|D^{j}\left(\Phi^{(n+1)}-\Phi^{(n)}\right)\right| \leq \frac{\left\|\Phi^{(n+1)}-\Phi^{(n)}\right\|_{\mathfrak{r}_{n+1}}}{\mathfrak{r}_{n+1}^{|j|}}<2 \varepsilon_{n}^{1 / 3} \cdot\left(\frac{32}{\mathfrak{r}_{0}} \tilde{q}_{n_{*}+n}^{2 \chi}\right)^{|j|}<\varepsilon_{n}^{1 / 6} .
$$

Therefore, $\Phi$ is $C^{\infty}$ in $\phi$ and hence $g_{\infty} \in C^{\infty}\left(\mathbb{T}^{2}, \mathbb{R}\right)$.

\section{Appendix: Proof of Proposition 4.1}

We solve the approximate equation of (4.1)

$$
e^{4 \pi i \varrho} h(\cdot+\alpha)-h+\mathcal{T}_{K}(\widetilde{g}(\varphi) h(\cdot+\alpha))=-\mathcal{T}_{K} f .
$$

Let

$$
\begin{array}{cc}
f(\varphi, \theta)=\sum_{l} f_{l}(\varphi) e^{2 \pi i\langle l, \theta\rangle}, \quad f_{l}(\varphi)=\sum_{k} \hat{f}_{l}(k) e^{2 \pi i k \varphi}, \\
h(\varphi, \theta)=\sum_{|l|<K} h_{l}(\varphi) e^{2 \pi i\langle l, \theta\rangle}, & h_{l}(\varphi)=\sum_{|k|<K-|l|} \hat{h}_{l}(k) e^{2 \pi i k \varphi} .
\end{array}
$$

The approximate equation (6.1) is equivalent to (for $|l|<K$ )

$$
\begin{aligned}
& e^{2 \pi i\left(\left\langle l, \alpha^{\prime}\right\rangle+2 \varrho\right)} h_{l}(\varphi+\tilde{\alpha})-h_{l}(\varphi) \\
& +e^{2 \pi i\left\langle l, \alpha^{\prime}\right\rangle} \mathcal{T}_{K-|l|}\left(\widetilde{g}(\varphi) h_{l}(\varphi+\tilde{\alpha})\right)=-\mathcal{T}_{K-|l|} f_{l}(\varphi) .
\end{aligned}
$$


For any fixed $l$, equation (6.2) can be viewed as a matrix equation

$$
\left(D_{l}+G_{l}\right) \bar{h}_{l}=-\bar{f}_{l}
$$

where

$$
\bar{h}_{l}=\left(\hat{h}_{l}(k)\right)_{|k|<K-|l|}^{T}, \quad \bar{f}_{l}=\left(\hat{f}_{l}(k)\right)_{|k|<K-|l|}^{T},
$$

and

$$
\begin{gathered}
D_{l}=\operatorname{diag}\left(e^{2 \pi i\left(\left\langle l, \alpha^{\prime}\right\rangle+2 \varrho+k \tilde{\alpha}\right)}-1:|k|<K-|l|\right), \\
G_{l}=\left(e^{2 \pi i\left(\left\langle l, \alpha^{\prime}\right\rangle+q \tilde{\alpha}\right) \hat{\widetilde{g}}}(p-q)\right)_{|p|,|q|<K-|l|} .
\end{gathered}
$$

If we denote

$$
\Omega_{l, r^{\prime}}=\operatorname{diag}\left(\cdots, e^{2 \pi|k| r^{\prime}}, \cdots\right)|k|<K-|l|
$$

for any $r^{\prime} \leq r$, then

$$
\Omega_{l, r^{\prime}}\left(D_{l}+G_{l}\right) \Omega_{l, r^{\prime}}^{-1} \Omega_{l, r^{\prime}} \bar{h}_{l}=-\Omega_{l, r^{\prime}} \bar{f}_{l} .
$$

Rewrite it as

$$
\left(D_{l}+\widetilde{G}_{l, r^{\prime}}\right) \widetilde{h}_{l, r^{\prime}}=-\widetilde{f}_{l, r^{\prime}},
$$

where

$$
\widetilde{G}_{l, r^{\prime}}=\Omega_{l, r^{\prime}} G_{l} \Omega_{l, r^{\prime}}^{-1}, \quad \widetilde{h}_{l, r^{\prime}}=\Omega_{l, r^{\prime}} \bar{h}_{l}, \quad \widetilde{f}_{l, r^{\prime}}=\Omega_{l, r^{\prime}} \bar{f}_{l},
$$

with the estimation

$$
\left\|\widetilde{G}_{l, r^{\prime}}\right\| \leq \frac{4\|\widetilde{g}\|_{r}}{1-e^{-2 \pi\left(r-r^{\prime}\right)}} \leq \frac{16 \pi\|g\|_{r}}{1-e^{-2 \pi\left(r-r^{\prime}\right)}},
$$

where the matrix norm is the 1-norm of a matrix. Moreover, one can check easily that

$$
\left\|\widetilde{h}_{l, r^{\prime}}\right\|=\sum_{|k|<K-|l|}\left|\hat{h}_{l}(k)\right| e^{2 \pi|k| r^{\prime}} .
$$

As $\varrho \in D_{\alpha}\left(\gamma^{\prime}, \tau^{\prime}\right)$, then for any $|k|+|l|<K$ we have $\left\|D_{l}^{-1}\right\| \leq \frac{K^{\tau^{\prime}}}{\gamma^{\prime}}$ and hence

$$
\left\|D_{l}^{-1} \widetilde{G}_{l, r-\sigma}\right\| \leq \frac{16 \pi K^{\tau^{\prime}} \eta}{\gamma^{\prime} \sigma}<\frac{1}{2}
$$

by (4.4) for $\sigma \leq \frac{1}{4}$. Thus, there is

$$
\left\|\left(I+D_{l}^{-1} \widetilde{G}_{l, r-\sigma}\right)^{-1}\right\|<2 .
$$

Then by (6.3), $\widetilde{h}_{l, r-\sigma}$ can be expressed as:

$$
\widetilde{h}_{l, r-\sigma}=-\left(I+D_{l}^{-1} \widetilde{G}_{l, r-\sigma}\right)^{-1} D_{l}^{-1} \widetilde{f}_{l, r-\sigma} .
$$


Let $\delta \geq \sigma$. We furthermore use (6.5) to obtain the estimation

$$
\begin{aligned}
& \|h(\varphi, \theta)\|_{r-\sigma, s-\delta} \\
& \leq \sum_{|k|+|l|<K}\left|\hat{h}_{l}(k)\right| e^{2 \pi|k|(r-\sigma)} e^{2 \pi|l|(s-\delta)} \\
& \leq \sum_{|l|<K}\left\|\widetilde{h}_{l, r-\sigma}\right\| e^{2 \pi|l|(s-\delta)} \\
& \leq \sum_{|l|<K}\left\|\left(I+D_{l}^{-1} \widetilde{G}_{l, r-\sigma}\right)^{-1}\right\| \cdot\left\|D_{l}^{-1}\right\| \cdot\left\|\widetilde{f}_{l, r-\sigma}\right\| e^{2 \pi|l|(s-\delta)} \\
& \leq \frac{2 K^{\tau^{\prime}}}{\gamma^{\prime}} \sum_{|l|<K|k|<K-|l|}\|f\|_{r, s} e^{-2 \pi|k| \sigma} e^{-2 \pi|l| \delta} \\
& \leq \frac{C_{0}(d) K^{\tau^{\prime}} \widetilde{\eta}}{\gamma^{\prime} \sigma^{d}} .
\end{aligned}
$$

Moreover, we can get the control of the error term:

$$
\begin{aligned}
\|\tilde{P}\|_{r-2 \sigma, s-2 \delta} & \leq\left\|\mathcal{R}_{K} f\right\|_{r-2 \sigma, s-2 \delta}+\left\|\mathcal{R}_{K}(\widetilde{g}(\varphi) h(\cdot+\alpha))\right\|_{r-2 \sigma, s-2 \delta} \\
& \leq C(d) K^{d} e^{-2 \pi K \sigma}\left(\|f\|_{r, s}+\|\widetilde{g}\|_{r} \cdot\|h\|_{r-\sigma, s-\delta}\right) \\
& \leq C_{1}(d) K^{d} \tilde{\eta}^{2}\left(1+\frac{K^{\tau^{\prime}}}{\gamma^{\prime} \sigma^{d}} \eta\right) .
\end{aligned}
$$

\section{ACKNowledgements}

QZ wants to thank Artur Avila for useful discussions. JW was supported by "the Fundamental Research Funds for the Central Universities", No. 30918011336, NNSF of China (Nos. 11601230, 11971233, 11701285), and the Natural Science Foundation of Jiangsu Province, China (No. BK20160816). XH was partially supported by NNSF of China (Grant 11371019, 11671395) and Self-Determined Research Funds of Central China Normal University (CCNU19QN078). QZ was support by NNSF grant (11671192, 11771077) and Nankai Zhide Foundation.

\section{REFERENCES}

[1] S. Amor, Hölder continuity of the rotation number for quasi-periodic co-cycles in $S L(2, \mathbb{R})$, Comm. Math. Phys. 287.2 (2009), 565-588.

[2] A. Avila, Global theory of one-frequency Schrödinger operators, Acta Math. 215.1 (2015), 1-54.

[3] A. Avila, Almost reducibility and absolute continuity I, arXiv preprint (2010), arXiv:1006.0704.

[4] A. Avila, KAM, Lyapunov exponents and the spectral dichotomy for one-frequency schrödinger operators. In preparation. 
[5] A. Avila, J. Bochi and D. Damanik, Cantor spectrum for Schrödinger operators with potentials arising from generalized skew-shifts, Duke Math. J. 146.2 (2009), 253-280.

[6] A. Avila, B. Fayad and R. Krikorian, A KAM scheme for $\operatorname{SL}(2, \mathbb{R})$ cocycles with Liouvillean frequencies, Geom. Funct. Anal. 21.5 (2011), 1001-1019.

[7] A. Avila and S. Jitomirskaya, Almost localization and almost reducibility, J. Eur. Math. Soc. 12.1 (2010), 93-131.

[8] A. Avila and S. Jitomirskaya, In preparation.

[9] A. Avila and R. Krikorian, Reducibility or non-uniform hyperbolicity for quasiperiodic Schrödinger cocycles, Ann. Math. 164 (2006), 911940.

[10] J. Bourgain, On the spectrum of lattice Schrödingr operators with deterministic potential II, J. Anal. Math. 88.1 (2002), 221-254.

[11] J. Bourgain, Positivity and continuity of the Lyapunov exponent for shifts on $T^{d}$ with arbitrary frequency vector and real analytic potential, J. Anal. Math. 96.1 (2005), 313-355.

[12] J. Bourgain and S. Jitomirskaya, Absolutely continuous spectrum for 1D quasiperiodic operators. Invent. math, 148.3 (2002), 453-463.

[13] K. Bjerklöv and R. Krikorian, Coexistence of ac and pp spectrum for kicked quasi-periodic potentials, arXiv:1901.04418v1 [math.DS] (2019).

[14] J. Bourgain and M. Goldstein, On nonperturbative localization with quasi-periodic potential, Ann. of Math. 152.3 (2000), 835-879.

[15] A. Cai, C. Chavaudret, J. You and Q. Zhou, Sharp hölder continuity of the Lyapunov exponent of finitely differentiable quasi-periodic cocycles, Math Z., 291.3-4 (2019), 931-958.

[16] D. Damanik, Schrödinger Operators with Dynamically Defined Potentials: A Survey, Ergodic Theory Dynam. Systems 37.6 (2017), 16811764 .

[17] E. I. Dinaburg and Y. G. Sinai, The one-dimensional Schrödinger equation with quasiperiodic potential, Funk. Anal. i Prilozen. 9.4 (1975), 279-289.

[18] P. Duarte and S. Klein, Topological obstructions to dominated splitting for ergodic translations on the higher dimensional torus, Discrete Contin. Dyn. Syst. A, 38.11 (2018), 5379-5387.

[19] H. Eliasson, Floquet solutions for the 1-dimensional quasi-periodic Schrödinger equation, Comm. Math. Phys. 146.3 (1992), 447-482.

[20] H. Eliasson, Personal communication.

[21] D. J. Gilbert and D. B. Pearson, On subordinacy and analysis of the spectrum of one-dimensional Schrödinger operators, J. Math. Anal. Appl. 128.1 (1987), 30-56.

[22] M. Goldstein and W. Schlag, Hölder continuity of the integrated density of states for quasiperiodic Schrödinger equations and averages of shifts of subharmonic functions, Ann. Math. 154.1 (2001), 155-203. 
[23] M. R. Herman, Une methode pour minorer les exposants de Lyapunov et quelques exemples montrant le charactere local d'un theoreme d'Arnold et de Moser sur le tore de dimension 2, Comment. Math. Helvetici, $\mathbf{5 8 . 3}$ (1983), 453-502.

[24] X. Hou and J. You, Almost reducibility and non-perturbative reducibility of quasi-periodic linear systems, Invent. Math. 190.1 (2012), 209-260.

[25] S. Jitomirskaya and C. A. Marx. Dynamics and spectral theory of quasiperiodic Schrödinger-type operators, Ergodic Theory Dynam. Systems, 37.8 (2017), 2353-2393.

[26] S. Kotani, Lyapunov indices determine absolutely continuous spectra of stationary random one dimensional Schrödinger operators, In: Northholland Mathematical Library, Vol. 32. Elsevier (1984), 225-247.

[27] R. Krikorian, Reducibility, differentiable rigidity and Lyapunov exponents for quasiperiodic cocycles on $\mathbb{T} \times S L(2, \mathbb{R})$, arXiv preprint (2004), arXiv:math.DS/0402333.

[28] R.Krikorian, J.Wang, J.You and Q. Zhou, Linearization of quasiperiodically forced circle flow beyond Brjuno condition, Comm. Math. Phys. 358.1 (2018), 81-100.

[29] Y. Last and B. Simon, Eigenfunctions, transfer matrices, and absolutely continuous spectrum of one-dimensional Schrödinger operators. Invent. Math. 135.2 (1999), 329-367.

[30] B. Simon, Kotani Theory for One Dimensional Stochastic Jacobi Matrices, Comm. Math. Phys. 89.2 (1983), 227-234.

[31] E. Sorets and T. Spencer, Positive Lyapunov exponents for Schr ödinger operators with quasi-periodic potentials, Comm. Math. Phys. 142.3 (1991), 543-566.

[32] J C. Yoccoz, Petits diviseurs en dimension 1, Astérisque, 1995.

[33] J. You and Q. Zhou, Phase transition and semi-global reducibility, Comm. Math. Phys. 330.3 (2014), 1095-1113.

School of Mathematic and Statics, Central China Normal University, Wuhan 430079, CHINA

E-mail address: hxj@mail.ccnu.edu.cn

Department of Mathematics, School of Science, Nanjing University of SciEnCE AND Technology, NANJing 210094, China

E-mail address: jingwang018@gmail.com, jing.wang@njust.edu.cn

Chern Institute of Mathematics and LPMC, Nankai University, Tianjin 300071, CHINA

E-mail address: qizhou628@gmail.com, qizhou@nankai.edu.cn 